\title{
Global Existence and Blow-Up for the Euler-Bernoulli Plate Equation with Variable Coefficients
}

\author{
Jianghao Hao and Jie Lan \\ School of Mathematical Sciences, Shanxi University, Taiyuan, Shanxi 030006, China \\ Correspondence should be addressed to Jianghao Hao; hjhao@sxu.edu.cn \\ Received 4 October 2014; Accepted 8 December 2014; Published 25 December 2014 \\ Academic Editor: Hugo Leiva
}

Copyright (C) 2014 J. Hao and J. Lan. This is an open access article distributed under the Creative Commons Attribution License, which permits unrestricted use, distribution, and reproduction in any medium, provided the original work is properly cited.

We prove the local existence, blow-up, global existence, and stability of solutions to the initial boundary value problem for EulerBernoulli plate equation with variable coefficients.

\section{Introduction}

Let $\Omega$ be a bounded domain in $R^{n}$ with smooth boundary and $\Gamma=\partial \Omega$. We will consider the following initial-boundary value problem:

$$
\begin{gathered}
u_{t t}+\mathscr{A}^{2} u-\gamma \mathscr{A} u_{t}+a\left|u_{t}\right|^{m-2} u_{t}=b|u|^{p-2} u, \\
x \in \Omega, \quad t \in(0, T), \\
u=\frac{\partial u}{\partial v_{\mathscr{A}}}=0, \quad x \in \Gamma, t \in(0, T), \\
u(x, 0)=u_{0}(x), \quad u_{t}(x, 0)=u_{1}(x), \quad x \in \bar{\Omega},
\end{gathered}
$$

where the constants $\gamma \geq 0, a \geq 0, b \geq 0, p \geq 2, m \geq 2$, and

$$
\mathscr{A} u=\sum_{i, j=1}^{n} \frac{\partial}{\partial x_{i}}\left(a_{i j}(x) \frac{\partial u}{\partial x_{j}}\right), \quad x \in R^{n}
$$

where $a_{i j}(x)=a_{j i}(x)$ are $C^{\infty}$ functions in $R^{n}$ satisfying

$$
\begin{aligned}
& \sum_{i, j=1}^{n} a_{i j}(x) \xi_{i} \xi_{j} \geq \lambda \sum_{i=1}^{n} \xi_{i}^{2}>0, \\
& \forall x \in R^{n}, \quad 0 \neq\left(\xi_{1}, \xi_{2}, \ldots, \xi_{n}\right)^{T} \in R^{n},
\end{aligned}
$$

where $\lambda$ is a positive constant. $\partial u / \partial v_{\mathscr{A}}=\sum_{i, j=1}^{n} a_{i j}(x)(\partial u /$ $\left.\partial x_{i}\right) v_{j}$ is the so-called conormal derivative and $\nu=\left(v_{1}, v_{2}, \ldots, v_{n}\right)$ is the unit normal of $\Gamma$ pointing towards the exterior of $\Omega$ and $v_{\mathscr{A}}=\mathscr{A} v$.

In physical terms the entries $a_{i j}(x)$ are related to coefficients of elasticity. Let $A(x)=\left(a_{i j}(x)\right)$ be an $n \times n$ matrix for $x \in R^{n}$, and let $x=\left(x_{1}, x_{2}, \ldots, x_{n}\right)$ be the natural coordinate system. For each $x \in R^{n}$, we define the inverse matrix of $A(x)$ by $\left(g_{i j}(x)\right)=\left(a_{i j}(x)\right)^{-1}$ and the inner product and norm over the tangent space $R_{x}^{n}=R^{n}$ by

$$
\begin{gathered}
g(X, Y)=\langle X, Y\rangle_{g}=\sum_{i, j=1}^{n} g_{i j} \alpha_{i} \beta_{j}, \\
|X|_{g}=\langle X, X\rangle_{g}^{1 / 2}, \quad X=\sum_{i=1}^{n} \alpha_{i} \frac{\partial}{\partial x_{i}}, \quad Y=\sum_{i=1}^{n} \beta_{i} \frac{\partial}{\partial x_{i}} .
\end{gathered}
$$

Then $\left(R^{n}, g\right)$ is a Riemannian manifold with metric $g$. Denote the gradient operator in metric $g$ by $\nabla_{g}$. Then we have

$$
\begin{gathered}
\nabla_{g} u=\sum_{i, j=1}^{n}\left(a_{i j}(x) \frac{\partial u}{\partial x_{i}}\right) \frac{\partial}{\partial x_{j}}, \\
\left|\nabla_{g} u\right|_{g}^{2}=\left\langle\nabla_{g} u, \nabla_{g} u\right\rangle_{g}=\sum_{i, j=1}^{n} a_{i j} \frac{\partial u}{\partial x_{i}} \frac{\partial u}{\partial x_{j}} .
\end{gathered}
$$

It is well known that the equation

$$
u_{t t}+b\left|u_{t}\right|^{m-2} u_{t}=\Delta u+a|u|^{p-2} u
$$


$(a, b \geq 0)$ with Dirichlet boundary condition has been studied by many authors. The interaction between the damping and the source term was first discussed by Levine $[1,2]$ in the linear damping case $m=2$. He showed that the solutions with negative initial energy blow up in finite time. This result was improved by Kalantarov and Ladyzhenskaya [3] to study more situations. Georgiev and Todorova [4] extended Levine's results to the nonlinear damping case $m \geq 2$ and $2<p \leq 2(n-1) /(n-2)$ if $n \geq 3$. In their work, the authors proved that global existence for arbitrary initial data when $p \leq m$, while blow-up result is showed when $p>m$ and the initial energy associated with the solutions is sufficiently negative. The blow-up result is extended by Levine and Serrin [5] to the case of negative initial energy and $m>1$ (and to abstract evolution problems). Hao et al. [6] studied the nonlinear wave equations with damping and source term. By using the energy compensation method, the authors proved the growth orders of the nonlinear strain term, the damping term, and the source term. They also prove that the solution to the problem exists globally and blows up, respectively, and the estimate for the blow-up time is given. For results of the same nature, we refer the reader to [5, 7-9] and the references therein. Chen and Zhou [10] considered a semilinear Petrovsky equation with damping and source terms. It was proved that the solution blows up in finite time if the positive initial energy satisfies a suitable condition and for the linear damping case the solution blows up in finite time even for vanishing initial energy. Zhou [11] gave an elegant argument to the solution to nonlinear evolution equations with vanishing initial energy. Yao [12] discussed the initial-boundary value problem for Euler-Bernoulli plate with variable coefficients and gave the observability inequality.

The Cauchy problem of (6) has been studied in the last decade; see [13-17]. Global existence for the Cauchy problem with arbitrarily chosen data in the energy space was proved by Todorova [15] for $p \leq m$. The case $m<p$ has a richer structure. The negativity of initial energy was used to prove blow-up in many papers. Zhou $[16,17]$ considered the Cauchy problem for a nonlinear wave equation with linear damping and source terms and showed that the solution blows up in finite time even for vanishing initial energy, and global existence and large time behavior also were discussed.

For the dynamics of marine risers with damping, Köhl [18] used the Lyapunov function technique to show that the zero solution of the system is stable. Kalantarov and Kurt [19] proved that the zero solution for the considered problem is globally asymptotically stable, and an estimate of the rate of decay of the solution is obtained.

The systems of plate equation have been studied by numerous authors; see [20-27]. Messaoudi [26] considered the semilinear Petrovsky equation with the damping and the source term and showed that the solution blows up in finite time if the growth order of damping term is larger than the growth order of source term and the energy is negative, whereas the solution is global if the growth order of damping term is not larger than the growth order of source term. Hoffmann and Rybka [24] studied the analyticity of the nonlinear term forces convergence of solutions for two equations of continuum mechanics. They showed that any solution with appropriate boundary and initial conditions has a limit as $t$ goes to infinity. Avalos et al. [20] are interested in the case of thermoelastic plate and they established stability of the rest state. Eden and Milani [21] discussed the exponential attractors for extensible beam equation. Note that [24] differs from $[20,21]$ because [24] included a viscous term $\Delta u_{t}$ which played an important role in their consideration. Guesmia [22] considered the system of plate equation with damping and proved that the solution decays exponentially if the damping term behaves like a linear function, whereas the decay is of a polynomial order otherwise. $\mathrm{Li}$ and $\mathrm{Wu}$ [25] discussed the plate stabilization problem with infinite damping and showed that the energy of the problem decays exponentially provided that the negative damping is sufficiently small. For the Cauchy problem of multidimensional generalized double dispersion equation, $\mathrm{Xu}$ and $\mathrm{Liu}$ [27] proved the existence and nonexistence of global weak solution by potential well method.

Our purpose in this paper is to give the local existence, blow-up, global existence, and stability of the solution to the initial-boundary value problem (1).

We will write $\|\cdot\|$ denoting the usual $L^{2}(\Omega)$ norm $\|\cdot\|_{L^{2}(\Omega)}$ and $\|\cdot\|_{p}$ denoting the usual $L^{p}(\Omega)$ norm $\|\cdot\|_{L^{p}(\Omega)}$ and denoting

$$
\int_{\Omega}\left\langle\nabla_{g} w, \nabla_{g} w\right\rangle_{g} d x=\left\|\left|\nabla_{g} w\right|_{g}\right\|^{2} .
$$

Let

$$
\begin{gathered}
W=\left\{u \in H^{2}(\Omega) \mid u=\frac{\partial u}{\partial v_{\mathscr{A}}}=0 \text { on } \Gamma\right\}, \\
\mathscr{H}=\left\{u \in H^{4}(\Omega) \cap W \mid \mathscr{A} u=\frac{\partial \mathscr{A} u}{\partial v_{\mathscr{A}}}=0 \text { on } \Gamma\right\} .
\end{gathered}
$$

By a weak solution of system (1) we mean a function $u$ : $[0, T) \rightarrow W$ satisfying

$$
\begin{gathered}
\frac{d}{d t} \int_{\Omega} u_{t} w d x+\int_{\Omega} \mathscr{A} u \mathscr{A} w d x+\gamma \int_{\Omega}\left\langle\nabla_{g} u_{t}, \nabla_{g} w\right\rangle_{g} d x \\
+a \int_{\Omega}\left|u_{t}\right|^{m-2} u_{t} w d x=b \int_{\Omega}|u|^{p-2} u w d x, \\
u(0)=u_{0}, \quad u_{t}(0)=u_{1},
\end{gathered}
$$

for any $w \in W$.

Our paper is organized as follows. In Section 2, we prove the local existence of the solution to the initial-boundary value problem (1). Section 3 contains the statements and the proof of the blow-up of the solution to problem (1) with $\gamma=0$. Section 4 is devoted to the blow-up result for problem (1) with $m=2$. In Section 5, we prove the global existence of the solution for problem (1). The last section is devoted to the asymptotic stability of the solution for problem (1).

\section{Local Existence Result}

In this section we establish a local existence result for the solution to problem (1) under suitable conditions on $m$ and $p$.

First, we give the following local existence result. 
Theorem 1 (local existence). Suppose that

$$
\begin{gathered}
p \geq 2, \quad \text { for } n \leq 4 \\
2 \leq p \leq \frac{2 n-2}{n-4}, \quad \text { for } n>4 \\
m \geq 2, \quad \text { for } n \leq 4 \\
2 \leq m \leq \frac{2 n}{n-4}, \quad \text { for } n>4 .
\end{gathered}
$$

Then for initial data $\left(u_{0}, u_{1}\right) \in W \times L^{2}(\Omega)$, there exists a unique weak solution of problem (1) satisfying

$$
\begin{aligned}
& u \in C([0, T], W), \\
& u_{t} \in C\left([0, T], L^{2}(\Omega)\right) \cap L^{m}(\Omega \times(0, T))
\end{aligned}
$$

for $T>0$ small enough.

\section{Proof.}

Step 1. For $v(x, t)$ given, we consider the local existence of the problem

$$
\begin{gathered}
u_{t t}+\mathscr{A}^{2} u-\gamma \mathscr{A} u_{t}+a\left|u_{t}\right|^{m-2} u_{t}=b|v|^{p-2} v, \\
x \in \Omega, \quad t \in(0, T), \\
u=\frac{\partial u}{\partial v_{\mathscr{A}}}=0, \quad x \in \Gamma, t \in(0, T), \\
u(x, 0)=u_{0}(x), \quad u_{t}(x, 0)=u_{1}(x), \quad x \in \bar{\Omega} .
\end{gathered}
$$

We take sequences $\left\{u_{0}^{\mu}\right\},\left\{u_{1}^{\mu}\right\} \in C_{0}^{\infty}(\Omega)$ to approximate $u_{0}$ and $u_{1}$, respectively, and take a sequence $\left\{v^{\mu}\right\} \in C\left([0, T], C_{0}^{\infty}(\Omega)\right)$ to approximate $v$. Then we consider the problems

$$
\begin{gathered}
u_{t t}^{\mu}+\mathscr{A}^{2} u^{\mu}-\gamma \mathscr{A} u_{t}^{\mu}+a\left|u_{t}^{\mu}\right|^{m-2} u_{t}^{\mu}=b\left|v^{\mu}\right|^{p-2} v^{\mu}, \\
x \in \Omega, \quad t \in(0, T), \\
u^{\mu}=\frac{\partial u^{\mu}}{\partial \nu_{\mathscr{A}}}=0, \quad x \in \Gamma, t \in(0, T), \\
u^{\mu}(x, 0)=u_{0}^{\mu}, \quad u_{t}^{\mu}(x, 0)=u_{1}^{\mu}, \quad x \in \bar{\Omega} .
\end{gathered}
$$

Using the same arguments as in [28], we get the existence of a sequence of unique solutions $\left\{\left(u^{\mu}, u_{t}^{\mu}\right)\right\}$ of (14) satisfying

$$
\begin{aligned}
& u^{\mu} \in L^{\infty}((0, T), \mathscr{H}), \\
& u_{t}^{\mu} \in L^{\infty}((0, T), W) \cap L^{m}(\Omega \times(0, T)), \\
& u_{t t}^{\mu} \in L^{\infty}\left((0, T), L^{2}(\Omega)\right) .
\end{aligned}
$$

For $R>0$ large and $T>0$, we let $Z_{T, R}$ denote the set of all functions $w$ which satisfy

$$
\begin{aligned}
& w \in C([0, T], W), \\
& w_{t} \in C\left([0, T], L^{2}(\Omega)\right) \cap L^{m}(\Omega \times(0, T)), \\
& w(x, 0)=u_{0}(x), \quad w_{t}(x, 0)=u_{1}(x), \quad x \in \bar{\Omega}, \\
& h(w(t)):=\left\|w_{t}\right\|_{C\left([0, T], L^{2}(\Omega)\right)}^{2}+\|\mathscr{A} w\|_{C\left([0, T], L^{2}(\Omega)\right)}^{2} \\
&+\left\|\left|\nabla_{g} w\right|_{g}\right\|_{L^{2}((0, T) \times \Omega)}^{2}+\left\|w_{t}\right\|_{L^{m}(\Omega \times(0, T))}^{2} \leq R^{2} .
\end{aligned}
$$

It follows from the trace theorem that $Z_{T, R}$ is nonempty if $R$ is sufficiently large and $T$ is small. For example, if we let

$$
w(x, t)=u_{0}(x) \cos t+u_{1}(x) \sin t,
$$

then $w \in Z_{T, R}$ for $R$ suitable large and $T$ small. In the present section, we always make this assumption.

Step 2. We proceed to show that the sequence $\left\{\left(u^{\mu}, u_{t}^{\mu}\right)\right\}$ is Cauchy in $Z_{T, R}$. For this aim, we set

$$
U:=u^{\mu}-u^{\nu}, \quad V:=v^{\mu}-v^{\nu},
$$

and then $U$ satisfies

$$
\begin{gathered}
U_{t t}+\mathscr{A}^{2} U-\gamma \mathscr{A} U_{t}+a\left|u_{t}^{\mu}\right|^{m-2} u_{t}^{\mu}-a\left|u_{t}^{\nu}\right|^{m-2} u_{t}^{\nu} \\
=b\left|v^{\mu}\right|^{p-2} v^{\mu}-b\left|v^{\nu}\right|^{p-2} v^{\nu}, \quad x \in \Omega, t \in(0, T), \\
U=\frac{\partial U}{\partial v_{\mathscr{A}}}=0, \quad x \in \Gamma, t \in(0, T), \\
U(x, 0)=U_{0}(x)=u_{0}^{\mu}-u_{0}^{\nu}, \quad U_{t}(x, 0)=U_{1}(x)=u_{1}^{\mu}-u_{1}^{\nu}, \\
x \in \bar{\Omega} .
\end{gathered}
$$

We multiply the equation of (19) by $U_{t}$ and integrate over $\Omega \times$ $(0, t)$; then it follows that

$$
\begin{aligned}
\int_{\Omega}\left(U_{t}^{2}+(\mathscr{A} U)^{2}\right) d x+2 \gamma \int_{0}^{t} \int_{\Omega}\left|\nabla_{g} U_{s}\right|_{g}^{2} d x d s \\
+2 a \int_{0}^{t} \int_{\Omega}\left(\left|u_{s}^{\mu}\right|^{m-2} u_{s}^{\mu}-\left|u_{s}^{\nu}\right|^{m-2} u_{s}^{\nu}\right)\left(u_{s}^{\mu}-u_{s}^{\nu}\right) d x d s \\
=\int_{\Omega}\left(U_{1}^{2}+\left(\mathscr{A} U_{0}\right)^{2}\right) d x \\
\quad+2 b \int_{0}^{t} \int_{\Omega}\left(\left|v^{\mu}\right|^{p-2} v^{\mu}-\left|v^{\nu}\right|^{p-2} v^{\nu}\right) U_{s} d x d s .
\end{aligned}
$$

To estimate from the above second term on the right side of (20), we use the inequality

$$
\begin{aligned}
& \left.|| v^{\mu}\right|^{p-2} v^{\mu}-\left|v^{\nu}\right|^{p-2} v^{\nu} \mid \\
& \quad \leq C_{1}\left|v^{\mu}-v^{\nu}\right|\left(\left|v^{\mu}\right|^{p-2}-\left|v^{\nu}\right|^{p-2}\right)
\end{aligned}
$$


for $v^{\mu}, v^{\nu} \in R$ and $p \geq 2$. Note that $C_{i}(i \geq 1)$ denote positive constants depending only on $p, m, \gamma, a, b$, and $\Omega$ rather than the initial data $\left(u_{0}, u_{1}\right)$ in this section. To estimate the second term on the left side of (20), we use the inequality

$$
\left(|w|^{m-2} w-|\widetilde{w}|^{m-2} \widetilde{w}\right)(w-\widetilde{w}) \geq C_{2}|w-\widetilde{w}|^{m}
$$

for $w, \widetilde{w} \in R$ and $m \geq 2$ with $w=u_{s}^{\mu}, \widetilde{w}=u_{s}^{\nu}$. Then (20)-(22) yield

$$
\begin{aligned}
\int_{\Omega}\left(U_{t}^{2}\right. & \left.+(\mathscr{A} U)^{2}\right) d x+2 \gamma \int_{0}^{t} \int_{\Omega}\left|\nabla_{g} U_{s}\right|_{g}^{2} d x d s \\
& +2 a C_{2} \int_{0}^{t} \int_{\Omega}\left|u_{s}^{\mu}-u_{s}^{\nu}\right|^{m} d x d s \\
\leq & \int_{\Omega}\left(U_{1}^{2}+\left(\mathscr{A} U_{0}\right)^{2}\right) d x \\
& +2 b C_{1} \int_{0}^{t} \int_{\Omega}\left|v^{\mu}-v^{\nu}\right|\left|U_{s}\right|\left(\left|v^{\mu}\right|^{p-2}-\left|v^{\nu}\right|^{p-2}\right) d x d s .
\end{aligned}
$$

From Hölder's inequality and the Sobolev embedding theorem the last term of (23) takes the form

$$
\begin{aligned}
I & :=2 b C_{1} \int_{0}^{t} \int_{\Omega}\left|v^{\mu}-v^{v}\right|\left|U_{s}\right|\left(\left|v^{\mu}\right|^{p-2}-\left|v^{\nu}\right|^{p-2}\right) d x d s \\
& \leq 2 b C_{3} \int_{0}^{t}\left\|U_{s}\right\|\|V\|_{2 n /(n-4)}\left[\left\|v^{\mu}\right\|_{n(p-2) / 2}^{p-2}+\left\|v^{\nu}\right\|_{n(p-2) / 2}^{p-2}\right] d s \\
& \leq 2 b C_{4} \int_{0}^{t}\left\|U_{s}\right\|\|\mathscr{A} V\|\left[\left\|\mathscr{A} v^{\mu}\right\|^{p-2}+\left\|\mathscr{A} v^{\nu}\right\|^{p-2}\right] d s \\
& \leq 4 b C_{4} R^{p-2} \int_{0}^{t}\left\|U_{s}\right\|\|\mathscr{A} V\| d s .
\end{aligned}
$$

Using Young's inequality, we have

$$
\begin{aligned}
I & \leq 2 b C_{4} R^{p-2} \int_{0}^{t}\left(\left\|U_{s}\right\|^{2}+\|\mathscr{A} V\|^{2}\right) d s \\
& \leq 2 b C_{4} R^{p-2} \int_{0}^{t}\left\|U_{s}\right\|^{2} d s+2 b C_{4} R^{p-2} T \max _{t \in[0, T]}\|\mathscr{A} V\|^{2} .
\end{aligned}
$$

Combining (23) and (25), we obtain, for any $t \in[0, T]$,

$$
\begin{aligned}
\left\|U_{t}\right\|^{2} & +\|\mathscr{A} U\|^{2}+2 \gamma\left\|\left|\nabla_{g} U_{t}\right|_{g}\right\|_{L^{2}((0, T) \times \Omega)}^{2} \\
& +2 a C_{2}\left\|U_{t}\right\|_{L^{m}((0, T) \times \Omega)}^{m} \\
\leq & \left\|U_{1}\right\|^{2}+\left\|\mathscr{A} U_{0}\right\|^{2}+2 b C_{4} R^{p-2} T \max _{t \in[0, T]}\|\mathscr{A} V\|^{2} \\
& +2 b C_{4} R^{p-2} \int_{0}^{t}\left\|U_{s}\right\|^{2} d s .
\end{aligned}
$$

It follows from (26) and Gronwall's inequality that, for any $t \in[0, T]$,

$$
\begin{aligned}
\| & U_{t}\left\|^{2}+\right\| \mathscr{A} U\left\|^{2}+2 \gamma\right\|\left|\nabla_{g} U_{t}\right|_{g} \|_{L^{2}((0, t) \times \Omega)}^{2} \\
& +2 a C_{2}\left\|U_{t}\right\|_{L^{m}((0, t) \times \Omega)}^{m} \\
\leq & {\left[\left\|U_{1}\right\|^{2}+\left\|\mathscr{A} U_{0}\right\|^{2}+2 b C_{4} R^{p-2} T \max _{t \in[0, T]}\|\mathscr{A} V\|^{2}\right] e^{2 b C_{4} R^{p-2} t} . }
\end{aligned}
$$

Furthermore, we have

$$
\begin{aligned}
& \max _{t \in[0, T]}\left\|U_{t}\right\|^{2}+\max _{t \in[0, T]}\|\mathscr{A} U\|^{2}+2 \gamma\left\|\left|\nabla_{g} U_{t}\right|_{g}\right\|_{L^{2}((0, T) \times \Omega)}^{2} \\
& \quad+2 a C_{2}\left\|U_{t}\right\|_{L^{m}((0, T) \times \Omega)}^{m} \\
& \leq\left[\left\|U_{1}\right\|^{2}+\left\|\mathscr{A} U_{0}\right\|^{2}+2 b C_{4} R^{p-2} T \max _{t \in[0, T]}\|\mathscr{A} V\|^{2}\right] e^{2 b C_{4} R^{p-2} T} .
\end{aligned}
$$

Since $\left\{v^{\mu}\right\},\left\{u_{0}^{\mu}\right\}$, and $\left\{u_{1}^{\mu}\right\}$ are Cauchy in $C([0, T] ; W), W$, and $L^{2}(\Omega)$, respectively, we conclude that $\left\{u^{\mu}\right\},\left\{u_{t}^{\mu}\right\},\left\{\left|\nabla_{g} u_{t}^{\mu}\right|_{g}\right\}$, and $\left\{u_{t}^{\mu}\right\}$ are Cauchy in $C([0, T] ; W), C\left([0, T] ; L^{2}(\Omega)\right)$, $L^{2}((0, T) \times \Omega)$, and $L^{m}((0, T) \times \Omega)$, respectively.

Step 3. We now prove that the limit $\left(u(x, t), u_{t}(x, t)\right)$ is a weak solution of (13).

To this end, we multiply equation of (14) by $w \in W$ and integrate over $\Omega$; then we obtain that

$$
\begin{gathered}
\frac{d}{d t} \int_{\Omega} u_{t}^{\mu} w d x+\int_{\Omega} \mathscr{A} u^{\mu} \mathscr{A} w d x+\gamma \int_{\Omega}\left\langle\nabla_{g} u_{t}^{\mu}, \nabla_{g} w\right\rangle_{g} d x \\
+a \int_{\Omega}\left|u_{t}^{\mu}\right|^{m-2} u_{t}^{\mu} w d x=b \int_{\Omega}\left|v^{\mu}\right|^{p-2} v^{\mu} w d x .
\end{gathered}
$$

As $\mu \rightarrow \infty$, the following hold:

$$
\begin{gathered}
\int_{\Omega} \mathscr{A} u^{\mu} \mathscr{A} w d x \rightarrow \int_{\Omega} \mathscr{A} u \mathscr{A} w d x, \quad \text { in } C([0, T]), \\
\int_{\Omega}\left|v^{\mu}\right|^{p-2} v^{\mu} w d x \rightarrow \int_{\Omega}|v|^{p-2} v w d x, \quad \text { in } C([0, T]), \\
\int_{\Omega}\left\langle\nabla_{g} u_{t}^{\mu}, \nabla_{g} w\right\rangle_{g} d x \longrightarrow \int_{\Omega}\left\langle\nabla_{g} u_{t}, \nabla_{g} w\right\rangle_{g} d x, \\
\text { in } C([0, T]),
\end{gathered}
$$$$
\int_{\Omega}\left|u_{t}^{\mu}\right|^{m-2} u_{t}^{\mu} w d x \longrightarrow \int_{\Omega}\left|u_{t}\right|^{m-2} u_{t} w d x, \quad \text { in } L^{1}((0, T)) .
$$

Then it follows that

$$
\int_{\Omega} u_{t} w d x\left(:=\lim _{\mu \rightarrow \infty} \int_{\Omega} u_{t}^{\mu} w d x\right)
$$

is an absolutely continuous function on $[0, T]$; thus for almost all $t \in[0, T],\left(u(x, t), u_{t}(x, t)\right)$ is a weak solution of 
problem (13). To prove uniqueness, we denote that $u^{\mu}, u^{\nu}$ are the corresponding solutions of problem (13) to $v^{\mu}, v^{\nu}$, respectively. Then $U=u^{\mu}-u^{\nu}$ satisfies

$$
\begin{aligned}
& \int_{\Omega}\left(U_{t}^{2}+(\mathscr{A} U)^{2}\right) d x+2 \gamma \int_{0}^{t} \int_{\Omega}\left|\nabla_{g} U_{s}\right|_{g}^{2} d x d s \\
& \quad+2 a C_{2} \int_{0}^{t} \int_{\Omega}\left|u_{s}^{\mu}-u_{s}^{v}\right|^{m} d x d s \\
& \leq 2 b C_{1} \int_{0}^{t} \int_{\Omega}\left|v^{\mu}-v^{\nu}\right|\left|U_{s}\right|\left(\left|v^{\mu}\right|^{p-2}-\left|v^{\nu}\right|^{p-2}\right) d x d s .
\end{aligned}
$$

This shows that $U=0$ for $v^{\mu}=v^{\nu}$. The uniqueness follows.

Step 4 . We denote by $\mathscr{K}$ the map which carries $v \in Z_{T, R}$ into $u$; that is,

$$
\mathscr{K} v=u \text {, }
$$

where $\left(u, u_{t}\right)$ is the solution of problem (13). We establish a priori estimate below to show that $\mathscr{K}$ maps $Z_{T, R}$ into itself if $R$ is sufficiently large and $T$ is sufficiently small relative to $R$. We then equip $Z_{T, R}$ with the complete metric $\rho$ defined by

$$
\rho(w, \bar{w})=\sup _{t \in[0, T]} h(w(t)-\bar{w}(t))
$$

and show that $\mathscr{K}$ is strict contraction if $T$ is sufficiently small. The contraction mapping principle thus implies that $\mathscr{K}$ has a unique fixed point which is obviously a solution to problem (1). For this purpose, we multiply the equation of (13) by $2 u_{t}$ and integrate over $\Omega \times(0, T)$ to get that

$$
\begin{aligned}
& \int_{\Omega}\left(u_{t}^{2}+(\mathscr{A} u)^{2}\right) d x+2 \gamma \int_{0}^{t} \int_{\Omega}\left|\nabla_{g} u_{s}\right|_{g}^{2} d x d s \\
& \quad+2 a \int_{0}^{t} \int_{\Omega}\left|u_{s}\right|^{m} d x d s \\
& =\int_{\Omega}\left(u_{1}^{2}+\left(\mathscr{A} u_{0}\right)^{2}\right) d x+2 b \int_{0}^{t} \int_{\Omega}|v|^{p-2} v u_{s} d x d s .
\end{aligned}
$$

From Hölder's inequality, it follows that, for $t \in[0, T]$,

$$
\begin{aligned}
& \int_{\Omega}\left(u_{t}^{2}+(\mathscr{A} u)^{2}\right) d x+2 \gamma \int_{0}^{t} \int_{\Omega}\left|\nabla_{g} u_{s}\right|_{g}^{2} d x d s \\
& \quad+2 a \int_{0}^{t} \int_{\Omega}\left|u_{s}\right|^{m} d x d s \\
& \leq \int_{\Omega}\left(u_{1}^{2}+\left(\mathscr{A} u_{0}\right)^{2}\right) d x+2 b \int_{0}^{t} \int_{\Omega}\left|u_{s}\right||v|^{p-1} d x d s
\end{aligned}
$$

$$
\begin{aligned}
\leq & \int_{\Omega}\left(u_{1}^{2}+\left(\mathscr{A} u_{0}\right)^{2}\right) d x+2 b C_{5} \int_{0}^{t}\left\|u_{s}\right\|\|\mathscr{A} v\|^{p-1} d s \\
\leq & \int_{\Omega}\left(u_{1}^{2}+\left(\mathscr{A} u_{0}\right)^{2}\right) d x+2 b C_{5} \max _{t \in[0, T]}\|\mathscr{A} v\|^{p-1} \int_{0}^{t}\left\|u_{s}\right\| d s \\
\leq & \int_{\Omega}\left(u_{1}^{2}+\left(\mathscr{A} u_{0}\right)^{2}\right) d x+2 b C_{5}\left[\max _{t \in[0, T]}\|\mathscr{A} v\|\right]^{p-1} \\
& \times \max _{t \in[0, T]}\left\|u_{t}\right\| \int_{0}^{t} d s \\
\leq & \int_{\Omega}\left(u_{1}^{2}+\left(\mathscr{A} u_{0}\right)^{2}\right) d x+2 b C_{5} T R^{p-1} \max _{t \in[0, T]}\left\|u_{t}\right\| .
\end{aligned}
$$

By choosing $R$ large enough and then $T$ sufficiently small, we obtain

$$
u \in Z_{T, R}
$$

This shows that $\mathscr{K}$ maps $Z_{T, R}$ into itself.

Step 5 . We verify that $\mathscr{K}$ is a contraction if $T$ is sufficiently small. Let $v, \bar{v} \in Z_{T, R}$, and set $u=\mathscr{K} v$ and $\bar{u}=\mathscr{K} \bar{v}$. Clearly, $U:=u-\bar{u}$ is the solution of the problem

$$
\begin{gathered}
U_{t t}+\mathscr{A}^{2} U-\gamma \mathscr{A} U_{t}+a\left|u_{t}\right|^{m-2} u_{t}-a\left|\bar{u}_{t}\right|^{m-2} \bar{u}_{t} \\
=b|v|^{p-2} v-b|\bar{v}|^{p-2} \bar{v} \quad x \in \Omega, t \in(0, T), \\
U=\frac{\partial U}{\partial v_{\mathscr{A}}}=0, \quad x \in \Gamma, t \in(0, T), \\
U(x, 0)=0, \quad U_{t}(x, 0)=0, \quad x \in \bar{\Omega} .
\end{gathered}
$$

Multiplying the equation of (38) by $2 U_{t}$ and integrating it over $\Omega$, we have

$$
\begin{aligned}
& \frac{d}{d t}\left[\left\|U_{t}\right\|^{2}+\|\mathscr{A} U\|^{2}+2 \gamma \int_{0}^{t} \int_{\Omega}\left|\nabla_{g} U_{s}\right|_{g}^{2} d x d s\right. \\
& \left.\quad+2 a \int_{0}^{t} \int_{\Omega}\left(\left|u_{s}\right|^{m-2} u_{s}-\left|\bar{u}_{s}\right|^{m-2} \bar{u}_{s}\right)\left(u_{s}-\bar{u}_{s}\right) d x d s\right] \\
& =2 b \int_{\Omega} U_{t}\left(|v|^{p-2} v-|\bar{v}|^{p-2} \bar{v}\right) d x .
\end{aligned}
$$

Noticing $U(x, 0)=U_{t}(x, 0)=0$ and using the same arguments as (20)-(28), we get the estimate

$$
\rho(u, \bar{u}) \leq 2 b C_{6} R^{p} T e^{2 b C_{6} R^{p-2} T} \rho(v, \bar{v}),
$$

where $C_{6}$ is a positive constant independent of $R$ and $T$. By choosing $T$ so small that

$$
2 b C_{6} R^{p} T e^{2 b C_{6} R^{p-2} T}<1,
$$

then the map $\mathscr{K}$ is a contraction. By the contraction mapping principle, the map $\mathscr{K}$ has a unique fixed point which is obviously a solution $u=\mathscr{K} v \in Z_{R, T}$. It is clear that $\left(u, u_{t}\right)$ is the desired solution of problem (1), and the proof of Theorem 1 is completed. 
In the following we denote the maximal existence time of the solution by $\left(T_{\max }=\right) T$ and the energy of system (1) by

$$
E(t)=\frac{1}{2}\left\|u_{t}\right\|^{2}+\frac{1}{2}\|\mathscr{A} u\|^{2}-\frac{b}{p}\|u\|_{p}^{p} .
$$

Multiplying the equation of (1) by $u_{t}$ and integrating over $\Omega$, we obtain, for $t \in[0, T)$,

$$
\frac{d}{d t} E(t)=-a\left\|u_{t}\right\|_{m}^{m}-\gamma\left\|\left|\nabla_{g} u_{t}\right|_{g}\right\|^{2} \leq 0
$$

Denote $B>0$ is the best embedding constant such that

$$
\|w\|_{p} \leq B\|\Delta w\|, \quad \text { for } w \in W \text {. }
$$

Next we present two lemmas which will be used in the following sections.

Lemma 2. Suppose that p satisfies (10); then one has

$$
\|u\|_{p}^{s} \leq\left(\frac{B^{2}}{\lambda}+1\right)\left[\|\mathscr{A} u\|^{2}+\|u\|_{p}^{p}\right]
$$

for $s \in[2, p]$.

Proof. From the fact that $\|u\|_{p} \leq B\|\Delta u\|$, we get

$$
\|u\|_{p}^{s} \leq\|u\|_{p}^{2} \leq B^{2}\|\Delta u\|^{2} \leq \frac{B^{2}}{\lambda}\|\mathscr{A} u\|^{2}
$$

if $\|u\|_{p} \leq 1$. Otherwise,

$$
\|u\|_{p}^{s} \leq\|u\|_{p}^{p}
$$

Lemma 2 follows from (46) and (47).

Lemma 3. Suppose that the conditions of Theorem 1 hold, and let $u(x, t)$ be the solution of problem (1) with the initial data satisfying $E(0)<E_{1}$ and $\left\|\mathscr{A} u_{0}\right\|>s_{1}$, where

$$
E_{1}:=\frac{p-2}{2 p} s_{1}^{2}, \quad s_{1}:=\left(\frac{\lambda^{p / 2}}{b B^{p}}\right)^{1 /(p-2)} .
$$

Then there exists $s_{2}>s_{1}$, such that

$$
\|\mathscr{A} u\| \geq s_{2} .
$$

Proof. By Sobolev-Poincare inequality and the property of the operator $\mathscr{A}$, we have

$$
\|u\|_{p}^{p} \leq B^{p}\|\Delta u\|^{p} \leq \frac{B^{p}}{\lambda^{p / 2}}\|\mathscr{A} u\|^{p}
$$

Thus we get

$$
\begin{aligned}
E_{1} & >E(0) \geq E(t) \geq \frac{1}{2}\|\mathscr{A} u\|^{2}-\frac{b}{p}\|u\|_{p}^{p} \\
& \geq \frac{1}{2}\|\mathscr{A} u\|^{2}-\frac{b B^{p}}{p \lambda^{p / 2}}\|\mathscr{A} u\|^{p}:=F(\|\mathscr{A} u\|),
\end{aligned}
$$

where

$$
F(s)=\frac{1}{2} s^{2}-\frac{b B^{p}}{p \lambda^{p / 2}} s^{p} .
$$

It is easy to verify that the function $F(s)$ has a maximum at $s_{1}=\left(\lambda^{p / 2} / b B^{p}\right)^{1 /(p-2)}$ and the maximum value is $E_{1}=$ $((p-2) / 2 p) s_{1}^{2}$. From the definition of $F(s)$, we see that $F(s)$ is increasing in $\left(0, s_{1}\right)$ and decreasing in $\left(s_{1},+\infty\right)$ and $F(s) \rightarrow$ $-\infty$ as $s \rightarrow+\infty$. From the assumptions $E(0)<E_{1}$ and $\left\|\mathscr{A} u_{0}\right\|>s_{1}$, we know that there exists $s_{2}>s_{1}$, such that

$$
\|\mathscr{A} u\| \geq s_{2}
$$

which completes the proof of Lemma 3.

\section{Blow-Up Result for $\gamma=0$}

In this section we establish the blow-up result for the following problem by the energy compensation method:

$$
\begin{gathered}
u_{t t}+\mathscr{A}^{2} u+a\left|u_{t}\right|^{m-2} u_{t}=b|u|^{p-2} u, \\
x \in \Omega, \quad t \in(0, T), \\
u=\frac{\partial u}{\partial v_{\mathscr{A}}}=0, \quad x \in \Gamma, t \in(0, T), \\
u(x, 0)=u_{0}(x), \quad u_{t}(x, 0)=u_{1}(x), \quad x \in \bar{\Omega} .
\end{gathered}
$$

Our technique of proof follows closely the argument of [7] with the modifications needed for our problem. Denoting the energy of system (54) by

$$
E(t)=\frac{1}{2}\left\|u_{t}\right\|^{2}+\frac{1}{2}\|\mathscr{A} u\|^{2}-\frac{b}{p}\|u\|_{p}^{p},
$$

multiplying the equation of (54) by $u_{t}$, and integrating over $\Omega$, we obtain, for $t \in[0, T)$,

$$
\frac{d}{d t} E(t)=-a\left\|u_{t}\right\|_{m}^{m}
$$

Next we give the blow-up result.

Theorem 4. Assume that the conditions of Theorem 1 hold, $m<p$, and $u(x, t)$ is the solution of (54) with the initial data 
$\left(u_{0}, u_{1}\right) \in W \times L^{2}(\Omega)$ satisfying either one of the following conditions:

(i) $E(0)<0$;

(ii) $0 \leq E(0)<E_{1}$ and $\left\|\mathscr{A} u_{0}\right\|>s_{1}$, in which

$$
E_{1}:=\frac{p-2}{2 p} s_{1}^{2}, \quad s_{1}:=\left(\frac{\lambda^{p / 2}}{b B^{p}}\right)^{1 /(p-2)}
$$

and $B$ is the embedding constant satisfying

$$
\|u\|_{p} \leq B\|\Delta u\|
$$

Then the solution $u(x, t)$ blows up in finite time.

Proof. In the following, we discuss two cases.

Case $1\left(0 \leq E(0)<E_{1}\right)$. We set

$$
H(t)=M-E(t),
$$

where $M>E(0)$ can be chosen later. Since

$$
H^{\prime}(t)=a\left\|u_{t}\right\|_{m}^{m} \geq 0
$$

and $H(0)=M-E(0)>0$, hence

$$
0<H(0) \leq H(t) .
$$

Next we define

$$
G(t)=H^{1-\alpha}(t)+\varepsilon \int_{\Omega} u u_{t} d x,
$$

where $\alpha, \varepsilon$ are to be determined positive constants and $\varepsilon$ is small enough. Integrating by parts and using the equation in (54), we obtain

$$
\begin{aligned}
G^{\prime}(t)= & (1-\alpha) H^{-\alpha}(t) H^{\prime}(t)+\varepsilon\left\|u_{t}\right\|^{2}-\varepsilon\|\mathscr{A} u\|^{2} \\
& -a \varepsilon \int_{\Omega}\left|u_{t}\right|^{m-2} u_{t} u d x+b \varepsilon\|u\|_{p}^{p} .
\end{aligned}
$$

To estimate the fourth term of (63), we use Young's inequality,

$$
\begin{aligned}
\int_{\Omega}\left|u_{t}\right|^{m-2} u_{t} u d x & \leq \int_{\Omega}|u|\left|u_{t}\right|^{m-1} d x \\
& \leq \frac{\delta^{m}}{m}\|u\|_{m}^{m}+\frac{m-1}{m} \delta^{-m /(m-1)}\left\|u_{t}\right\|_{m}^{m},
\end{aligned}
$$

where $\delta$ can be time dependent, since the integral is taken over the $x$ variable. Take $\delta$ so that

$$
\delta^{-m /(m-1)}=k H^{-\alpha}(t),
$$

for sufficiently large $k$ to be specified later. Hence together with the definitions of $E(t)$ and $H(t)$, it follows that

$$
\begin{aligned}
G^{\prime}(t) \geq & {\left[1-\alpha-\frac{m-1}{m} \varepsilon k\right] H^{-\alpha}(t) H^{\prime}(t) } \\
& +\varepsilon\left(1+\frac{p}{2}\right)\left\|u_{t}\right\|^{2}+\varepsilon\left(\frac{p}{2}-1\right)\|\mathscr{A} u\|^{2} \\
& -\frac{a \varepsilon k^{1-m}}{m} H^{\alpha(m-1)}(t)\|u\|_{m}^{m}+\varepsilon p H(t)-\varepsilon p M .
\end{aligned}
$$

Furthermore, by Lemma 3 we have

$$
\begin{aligned}
\|\mathscr{A} u\|^{2} & =\frac{s_{2}^{2}-s_{1}^{2}}{s_{2}^{2}}\|\mathscr{A} u\|^{2}+\frac{s_{1}^{2}}{s_{2}^{2}}\|\mathscr{A} u\|^{2} \\
& \geq \frac{s_{2}^{2}-s_{1}^{2}}{s_{2}^{2}}\|\mathscr{A} u\|^{2}+s_{1}^{2} .
\end{aligned}
$$

Thus it follows from (66) and (67) that

$$
\begin{aligned}
G^{\prime}(t) \geq & {\left[1-\alpha-\frac{m-1}{m} \varepsilon k\right] H^{-\alpha}(t) H^{\prime}(t) } \\
& +\varepsilon\left(1+\frac{p}{2}\right)\left\|u_{t}\right\|^{2}+\varepsilon\left(\frac{p}{2}-1\right) \frac{s_{2}^{2}-s_{1}^{2}}{s_{2}^{2}}\|\mathscr{A} u\|^{2} \\
& -\frac{a \varepsilon k^{1-m}}{m} H^{\alpha(m-1)}(t)\|u\|_{m}^{m}+\varepsilon p H(t) \\
& +\varepsilon\left(\frac{p}{2}-1\right) s_{1}^{2}-\varepsilon p M .
\end{aligned}
$$

By taking $M=E_{1}$, we obtain

$$
\begin{aligned}
G^{\prime}(t) \geq & {\left[1-\alpha-\frac{m-1}{m} \varepsilon k\right] H^{-\alpha}(t) H^{\prime}(t) } \\
& +\varepsilon\left(1+\frac{p}{2}\right)\left\|u_{t}\right\|^{2}+\varepsilon\left(\frac{p}{2}-1\right) \frac{s_{2}^{2}-s_{1}^{2}}{s_{2}^{2}}\|\mathscr{A} u\|^{2} \\
& -\frac{a \varepsilon k^{1-m}}{m} H^{\alpha(m-1)}(t)\|u\|_{m}^{m}+\varepsilon p H(t) .
\end{aligned}
$$

Note that, from the definition of $E(t)$ and (48), we obtain

$$
\begin{aligned}
0 & <H(0) \leq H(t)=M-E(t) \leq M-\frac{1}{2}\|\mathscr{A} u\|^{2}+\frac{b}{p}\|u\|_{p}^{p} \\
& <M-\frac{1}{2} s_{2}^{2}+\frac{b}{p}\|u\|_{p}^{p} \leq \frac{b}{p}\|u\|_{p}^{p} .
\end{aligned}
$$

Thus by (70) and the inequality

$$
\|u\|_{m}^{m} \leq\|u\|_{p}^{m}
$$

we obtain

$$
H^{\alpha(m-1)}(t)\|u\|_{m}^{m} \leq\left(\frac{b}{p}\right)^{\alpha(m-1)}\|u\|_{p}^{\alpha p(m-1)+m} .
$$

Selecting $\alpha \in(0,(p-m) / p(m-1)]$, then together with Lemma 2, we get that

$$
H^{\alpha(m-1)}(t)\|u\|_{m}^{m} \leq\left(\frac{b}{p}\right)^{\alpha(m-1)}\left(\frac{B^{2}}{\lambda}+1\right)\left[\|\mathscr{A} u\|^{2}+\|u\|_{p}^{p}\right] .
$$


Combining (69) and (73), we have

$$
\begin{aligned}
G^{\prime}(t) \geq & {\left[1-\alpha-\frac{m-1}{m} \varepsilon k\right] H^{-\alpha}(t) H^{\prime}(t) } \\
& +\varepsilon\left(1+\frac{p}{2}\right)\left\|u_{t}\right\|^{2}+\varepsilon\left(\frac{p}{2}-1\right) \frac{s_{2}^{2}-s_{1}^{2}}{s_{2}^{2}}\|\mathscr{A} u\|^{2} \\
& -\frac{a \varepsilon k^{1-m}}{m}\left(\frac{b}{p}\right)^{\alpha(m-1)}\left(\frac{B^{2}}{\lambda}+1\right)\left[\|\mathscr{A} u\|^{2}+\|u\|_{p}^{p}\right] \\
& +\varepsilon p H(t) .
\end{aligned}
$$

From (74) and the equality

$$
H(t)=M+\frac{b}{p}\|u\|_{p}^{p}-\frac{1}{2}\left\|u_{t}\right\|^{2}-\frac{1}{2}\|\mathscr{A} u\|^{2},
$$

we obtain

$$
\begin{aligned}
G^{\prime}(t) \geq & {\left[1-\alpha-\frac{m-1}{m} \varepsilon k\right] H^{-\alpha}(t) H^{\prime}(t) } \\
+ & \varepsilon\left(1+\frac{p}{2}-\frac{p-2}{4} \frac{s_{2}^{2}-s_{1}^{2}}{s_{2}^{2}}\right)\left\|u_{t}\right\|^{2} \\
+ & \varepsilon\left[\frac{p-2}{4} \frac{s_{2}^{2}-s_{1}^{2}}{s_{2}^{2}}\right. \\
& \left.-\frac{a}{m} k^{1-m}\left(\frac{b}{p}\right)^{\alpha(m-1)}\left(\frac{B^{2}}{\lambda}+1\right)\right]\|\mathscr{A} u\|^{2} \\
+ & {\left[\frac{b(p-2)}{2 p} \frac{s_{2}^{2}-s_{1}^{2}}{s_{2}^{2}}\right.} \\
& \left.\quad-\frac{a}{m} k^{1-m}\left(\frac{b}{p}\right)^{\alpha(m-1)}\left(\frac{B^{2}}{\lambda}+1\right)\right]\|u\|_{p}^{p} \\
+ & {\left[p-\frac{(p-2)}{2} \frac{s_{2}^{2}-s_{1}^{2}}{s_{2}^{2}}\right] H(t)+\varepsilon \frac{p-2}{2} \frac{s_{2}^{2}-s_{1}^{2}}{s_{2}^{2}} M . }
\end{aligned}
$$

Then we can choose $k$ large enough such that the coefficients of $\|\mathscr{A} u\|^{2}$ and $\|u\|_{p}^{p}$ in (76) are strictly positive, and hence we have

$$
\begin{aligned}
G^{\prime}(t) \geq & {\left[1-\alpha-\frac{m-1}{m} \varepsilon k\right] H^{-\alpha}(t) H^{\prime}(t) } \\
& +\varepsilon \beta\left[\left\|u_{t}\right\|^{2}+\|\mathscr{A} u\|^{2}+\|u\|_{p}^{p}+H(t)\right],
\end{aligned}
$$

where $\beta>0$ is the minimum of these coefficients. For fixed $k$, we pick $\varepsilon$ small enough such that $1-\alpha-((m-1) / m) \varepsilon k \geq 0$ and

$$
G(0)=H^{1-\alpha}(0)+\varepsilon \int_{\Omega} u_{0} u_{1} d x>0 .
$$

Hence

$$
G^{\prime}(t) \geq \varepsilon \beta\left[\left\|u_{t}\right\|^{2}+\|\mathscr{A} u\|^{2}+\|u\|_{p}^{p}+H(t)\right] .
$$

Thus for $t \geq 0, G(t)$ is a nondecreasing function, and we have, for all $t \geq 0$,

$$
G(t) \geq G(0)>0 .
$$

Here we estimate $G^{1 /(1-\alpha)}(t)$ from above. In the following of this section, we denote by $C$ the general positive constant which depends on $|\Omega|, p, \alpha$, and $H(0)$.

We use Hölder's inequality to estimate the term

$$
\left|\int_{\Omega} u u_{t} d x\right| \leq\|u\|\left\|u_{t}\right\| \leq C\|u\|_{p}\left\|u_{t}\right\|
$$

which implies

$$
\left|\int_{\Omega} u u_{t} d x\right|^{1 /(1-\alpha)} \leq C\|u\|_{p}^{1 /(1-\alpha)}\left\|u_{t}\right\|^{1 /(1-\alpha)} .
$$

Using Young's inequality

$$
X Y \leq \frac{\delta^{\mu}}{\mu} X^{\mu}+\frac{\delta^{-\theta}}{\theta} Y^{\theta}, \quad X, Y \geq 0, \quad \forall \delta>0, \frac{1}{\mu}+\frac{1}{\theta}=1,
$$

with $\delta=1, \mu=2(1-\alpha) /(1-2 \alpha)$, and $\theta=2(1-\alpha)$, we have

$$
\left|\int_{\Omega} u u_{t} d x\right|^{1 /(1-\alpha)} \leq C\left[\|u\|_{p}^{2 /(1-2 \alpha)}+\left\|u_{t}\right\|^{2}\right] .
$$

By choosing

$$
0<\alpha \leq \min \left\{\frac{p-2}{2 p}, \frac{p-m}{p(m-1)}\right\},
$$

which implies $2 /(1-2 \alpha) \leq p$, then using (70) and the inequality

$$
z^{\nu} \leq z+1 \leq\left(1+\frac{1}{a}\right)(z+a), \quad \forall z \geq 0, a>0,0<v \leq 1,
$$

with $z=\|u\|_{p}^{p}$ and $a=H(0)$, we deduce

$$
\left|\int_{\Omega} u u_{t} d x\right|^{1 /(1-\alpha)} \leq C\left(1+\frac{1}{H(0)}\right)\left(\|u\|_{p}^{p}+\left\|u_{t}\right\|^{2}+H(t)\right) .
$$

Consequently, we have, for all $t>0$,

$$
\begin{aligned}
G^{1 /(1-\alpha)}(t) & =\left(H^{1-\alpha}(t)+\varepsilon \int_{\Omega} u u_{t} d x\right)^{1 /(1-\alpha)} \\
& \leq 2^{1 /(1-\alpha)}\left(H(t)+\left|\int_{\Omega} u u_{t} d x\right|^{1 /(1-\alpha)}\right) \\
& \leq C\left[H(t)+\|u\|_{p}^{p}+\left\|u_{t}\right\|^{2}\right] .
\end{aligned}
$$

It follows from (79) and (88) that

$$
G^{\prime}(t) \geq C G^{1 /(1-\alpha)}(t) .
$$


A simple integration of $(89)$ over $(0, t)$ then yields

$$
G^{\alpha /(1-\alpha)}(t) \geq \frac{1}{G^{-\alpha /(1-\alpha)}(0)-C \alpha t /(1-\alpha)} .
$$

Therefore (90) shows that $G(t)$ blows up in a finite time $T^{*}$ given by the estimate

$$
T^{*} \leq \frac{1-\alpha}{C \alpha G^{\alpha /(1-\alpha)}(0)} .
$$

So the solution $u(x, t)$ blows up in a certain finite time.

Case $2(E(0)<0)$. We can take $M=0$ in the definition of $H(t)$; that is,

$$
H(t)=-E(t)
$$

Then we can get our result by the same arguments as in Case 1. This completes the proof of Theorem 4 .

Remark 5. The earliest blow-up time $T^{*}$ can be estimated by

$$
T^{*} \leq \frac{1-\alpha}{C \alpha G^{\alpha /(1-\alpha)}(0)}
$$

and the larger the $G(0)$ is, the quicker the blow-up takes place.

\section{Blow-Up Result for $m=2$}

In this section we discuss the blow-up result for the following problem:

$$
\begin{gathered}
u_{t t}+\mathscr{A}^{2} u-\gamma \mathscr{A} u_{t}+a u_{t}=b|u|^{p-2} u, \\
x \in \Omega, \quad t \in(0, T), \\
u=\frac{\partial u}{\partial v_{\mathscr{A}}}=0, \quad x \in \Gamma, t \in(0, T), \\
u(x, 0)=u_{0}(x), \quad u_{t}(x, 0)=u_{1}(x), \quad x \in \bar{\Omega},
\end{gathered}
$$

with the concavity method; see Levine $[1,2]$.

Denoting the energy of system (94) by

$$
E(t)=\frac{1}{2}\left\|u_{t}\right\|^{2}+\frac{1}{2}\|\mathscr{A} u\|^{2}-\frac{b}{p}\|u\|_{p}^{p}
$$

and multiplying the equation of (94) by $u_{t}$ and integrating over $\Omega$, we obtain, for $t \in[0, T)$,

$$
\frac{d}{d t} E(t)=-a\left\|u_{t}\right\|^{2}-\gamma\left\|\left|\nabla_{g} u_{t}\right|_{g}\right\|^{2}
$$

Our result is as follows.

Theorem 6. Suppose $p$ satisfies (10), $u(x, t)$ is the solution of problem (94), and the initial data $\left(u_{0}, u_{1}\right) \in W \times L^{2}(\Omega)$ satisfies either one of the following conditions:

(i) $E(0)<0$;

(ii) $E(0)=0$ and $\int_{\Omega} u_{0} u_{1} d x>0$; (iii) $0<E(0)<E_{1}$ and $\left\|\mathscr{A} u_{0}\right\|>s_{1}$, where

$$
E_{1}:=\frac{p-2}{2 p} s_{1}^{2}, \quad s_{1}:=\left(\frac{\lambda^{p / 2}}{b B^{p}}\right)^{1 /(p-2)},
$$

and $B$ is the embedding constant satisfying

$$
\|u\|_{p} \leq B\|\Delta u\|
$$

Then the solution $u(x, t)$ blows up in finite time $T$, and

$$
\lim _{t \rightarrow T^{-}}\left(\|u(t)\|^{2}+\left\|\left|\nabla_{g} u\right|_{g}\right\|^{2}\right)=+\infty
$$

Proof. Define

$$
\begin{aligned}
L(t)= & \|u(t)\|^{2}+a \int_{0}^{t}\|u(s)\|^{2} d s \\
& +\gamma \int_{0}^{t}\left\|\left|\nabla_{g} u(s)\right|_{g}\right\|^{2} d s+a\left(T_{0}-t\right)\left\|u_{0}\right\|^{2} \\
& +\gamma\left(T_{0}-t\right)\left\|\left|\nabla_{g} u_{0}\right|_{g}\right\|^{2}+r(t+\tau)^{2}, \quad t \in\left[0, T_{0}\right],
\end{aligned}
$$

where $T_{0}, r$, and $\tau$ are positive constants which are specified latter.

It is not difficult to see that $L(t)>0$ for all $t \in\left[0, T_{0}\right]$. Furthermore, we have

$$
\begin{aligned}
L^{\prime}(t)= & 2 \int_{\Omega} u u_{t} d x+a\|u\|^{2}-a\left\|u_{0}\right\|^{2}+\gamma\left\|\left|\nabla_{g} u\right|_{g}\right\|^{2} \\
& -\gamma\left\|\left|\nabla_{g} u_{0}\right|_{g}\right\|^{2}+2 r(t+\tau) \\
= & 2 \int_{\Omega} u u_{t} d x+2 a \int_{0}^{t} \int_{\Omega} u u_{s}(x, s) d x d s \\
& +2 \gamma \int_{0}^{t} \int_{\Omega}\left\langle\nabla_{g} u(x, s), \nabla_{g} u_{s}(x, s)\right\rangle_{g} d x d s \\
& +2 r(t+\tau), \\
L^{\prime \prime}(t)= & 2\left\|u_{t}\right\|^{2}-2\|\mathscr{A} u\|^{2}+2 b\|u\|_{p}^{p}+2 r .
\end{aligned}
$$


10

Journal of Function Spaces

Combining (100) and (101), we obtain

$$
\begin{gathered}
L L^{\prime \prime}-\frac{p+2}{4}\left(L^{\prime}\right)^{2} \\
=2 L\left[\left\|u_{t}\right\|^{2}-\|\mathcal{A} u\|^{2}+b\|u\|_{p}^{p}+r\right] \\
+(p+2)\left\{\left[\|u\|^{2}+a \int_{0}^{t}\|u(s)\|^{2} d s\right.\right. \\
\left.+\gamma \int_{0}^{t}\left\|\left|\nabla_{g} u(s)\right|_{g}\right\|^{2} d s+r(t+\tau)^{2}\right] \\
\times\left[\left\|u_{t}\right\|^{2}+a \int_{0}^{t}\left\|u_{s}\right\|^{2} d s\right. \\
\left.+\gamma \int_{0}^{t}\left\|\left|\nabla_{g} u_{s}\right|_{g}\right\|^{2} d s+r\right] \\
\quad\left[\int_{\Omega} u u_{t} d x+a \int_{0}^{t} \int_{\Omega} u u_{s} d x d s\right. \\
\left.+\gamma \int_{0}^{t}\left\|\left|\nabla_{g} u(s)\right|_{g}\right\|^{2} d s+r(t+\tau)^{2}\right] \\
+\left(p \int _ { 0 } ^ { t } \int _ { \Omega } \left\langle\left.u_{s}\left\|^{2} d s+\gamma \int_{0}^{t}\right\| \nabla_{g} u_{s}\right|_{g} \|_{s}^{2} d s+r d x d s\right.\right. \\
\left.+r(t+\tau)]_{g}^{2}\right\}
\end{gathered}
$$

It follows from the fact

$$
\begin{gathered}
\left(\int_{\Omega} u u_{t} d x+a \int_{0}^{t} \int_{\Omega} u u_{s} d x d s\right. \\
\left.+\gamma \int_{0}^{t} \int_{\Omega}\left\langle\nabla_{g} u, \nabla_{g} u_{s}\right\rangle_{g} d x d s+r(t+\tau)\right)^{2} \\
\leq\left(\|u\|^{2}+a \int_{0}^{t}\|u(s)\|^{2} d s\right. \\
\left.+\gamma \int_{0}^{t}\left\|\left|\nabla_{g} u\right|_{g}\right\|^{2} d s+r(t+\tau)^{2}\right) \\
\times\left(\left\|u_{t}\right\|^{2}+a \int_{0}^{t}\left\|u_{s}\right\|^{2} d s\right. \\
\left.+\gamma \int_{0}^{t}\left\|\left|\nabla_{g} u_{s}\right|_{g}\right\|^{2} d s+r\right)
\end{gathered}
$$

(103) that

$$
\begin{aligned}
& L L^{\prime \prime}-\frac{p+2}{4}\left(L^{\prime}\right)^{2} \\
& \geq 2 L\left[\left\|u_{t}\right\|^{2}-\|\mathscr{A} u\|^{2}+b\|u\|_{p}^{p}+r\right] \\
& \quad-(p+2)\left[\|u\|^{2}+a \int_{0}^{t}\|u(s)\|^{2} d s\right. \\
& \left.\quad+\gamma \int_{0}^{t}\left\|\left|\nabla_{g} u(s)\right|_{g}\right\|^{2} d s+r(t+\tau)^{2}\right] \\
& \quad \times\left[\left\|u_{t}\right\|^{2}+a \int_{0}^{t}\left\|u_{s}\right\|^{2} d s+\gamma \int_{0}^{t}\left\|\left|\nabla_{g} u_{s}\right|_{g}\right\|^{2} d s+r\right] .
\end{aligned}
$$

Noticing that

$$
\begin{gathered}
L(t)-\|u(t)\|^{2}-a \int_{0}^{t}\|u\|^{2} d s-\gamma \int_{0}^{t}\left\|\left|\nabla_{g} u\right|_{g}\right\|^{2} d s-r(t+\tau)^{2} \\
=a\left(T_{0}-t\right)\left\|u_{0}\right\|^{2}+\gamma\left(T_{0}-t\right)\left\|\left|\nabla_{g} u_{0}\right|_{g}\right\|^{2},
\end{gathered}
$$

then we get that

$$
\begin{aligned}
L(t) L^{\prime \prime}(t) & -\frac{p+2}{4}\left(L^{\prime}(t)\right)^{2} \\
\geq & 2 L(t)\left[\left\|u_{t}\right\|^{2}-\|\mathscr{A} u\|^{2}+b\|u\|_{p}^{p}+r\right] \\
& +(p+2)\left[a\left(T_{0}-t\right)\left\|u_{0}\right\|^{2}+\gamma\left(T_{0}-t\right)\left\|\left|\nabla_{g} u_{0}\right|_{g}\right\|^{2}\right] \\
& \times\left[\left\|u_{t}\right\|^{2}+a \int_{0}^{t}\left\|u_{s}\right\|^{2} d s+\gamma \int_{0}^{t}\left\|\left|\nabla_{g} u_{s}\right|_{g}\right\|^{2} d s+r\right] \\
& -(p+2) L(t)\left[\left\|u_{t}\right\|^{2}+a \int_{0}^{t}\left\|u_{s}\right\|^{2} d s\right. \\
& \left.+\gamma \int_{0}^{t}\left\|\left|\nabla_{g} u_{s}\right|_{g}\right\|^{2} d s+r\right]
\end{aligned}
$$

$$
\begin{aligned}
& \geq 2 L(t)\left[-\frac{p}{2}\left\|u_{t}\right\|^{2}-\|\mathscr{A} u\|^{2}+b\|u\|_{p}^{p}\right. \\
& \quad-\frac{a(p+2)}{2} \int_{0}^{t}\left\|u_{s}\right\|^{2} d s \\
& \left.\quad-\frac{\gamma(p+2)}{2} \int_{0}^{t}\left\|\left|\nabla_{g} u_{s}\right|_{g}\right\|^{2} d s-\frac{p}{2} r\right] \\
& :=2 L(t) Q(t),
\end{aligned}
$$
where $Q(t)$ is defined by

$$
\begin{aligned}
Q(t):= & -\frac{p}{2}\left\|u_{t}\right\|^{2}-\|\mathscr{A} u\|^{2}+b\|u\|_{p}^{p}-\frac{a(p+2)}{2} \int_{0}^{t}\left\|u_{s}\right\|^{2} d s \\
& -\frac{\gamma(p+2)}{2} \int_{0}^{t}\left\|\left|\nabla_{g} u_{s}\right|_{g}\right\|^{2} d s-\frac{p}{2} r .
\end{aligned}
$$


By the definition of $E(t)$, we may also write

$$
\begin{aligned}
Q(t)= & -p E(t)+\frac{p-2}{2}\|\mathscr{A} u\|^{2}-\frac{a(p+2)}{2} \int_{0}^{t}\left\|u_{s}\right\|^{2} d s \\
& -\frac{\gamma(p+2)}{2} \int_{0}^{t}\left\|\left|\nabla_{g} u_{s}\right|_{g}\right\|^{2} d s-\frac{p r}{2} .
\end{aligned}
$$

From (96), we find

$$
E(t)=E(0)-a \int_{0}^{t}\left\|u_{s}\right\|^{2} d s-\gamma \int_{0}^{t}\left\|\left|\nabla_{g} u_{s}\right|_{g}\right\|^{2} d s .
$$

Then we obtain

$$
\begin{aligned}
Q(t)= & -p E(0)+\frac{p-2}{2}\|\mathscr{A} u\|^{2}+\frac{a(p-2)}{2} \int_{0}^{t}\left\|u_{s}\right\|^{2} d s \\
& +\frac{\gamma(p-2)}{2} \int_{0}^{t}\left\|\left|\nabla_{g} u_{s}\right|_{g}\right\|^{2} d s-\frac{p r}{2} .
\end{aligned}
$$

In the following, we discuss three cases.

Case $1(E(0)<0)$. By taking $r=-2 E(0)>0$, we have

$$
\begin{gathered}
Q(t) \geq-p\left(E(0)+\frac{r}{2}\right)=0, \\
L(0)>0 .
\end{gathered}
$$

Furthermore, we have

$$
L(t) L^{\prime \prime}(t)-\frac{p+2}{4}\left(L^{\prime}(t)\right)^{2} \geq 0, \quad t \in\left[0, T_{0}\right] .
$$

Denoting $y(t)=L(t)^{-(p-2) / 4}$, we obtain that

$$
\begin{aligned}
y^{\prime \prime}(t) & =-\frac{p-2}{4} L(t)^{-(p+6) / 4}\left[L(t) L^{\prime \prime}(t)-\frac{p+2}{4}\left(L^{\prime}(t)\right)^{2}\right] \\
& \leq 0, \quad t \in\left[0, T_{0}\right] .
\end{aligned}
$$

We can select suitable positive constants $\tau$ and $T$ such that

$$
L^{\prime}(0)>0, \quad 0<\frac{L(0)}{L^{\prime}(0)} \leq \frac{p-2}{4} T,
$$

and then we have

$$
L(t) \geq\left(\frac{4 L^{(p+2) / 4}(0)}{4 L(0)-(p-2) L^{\prime}(0) t}\right)^{4 /(p-2)},
$$

for some $t>0$. Moreover, there exists a $T>0$ such that $T \leq$ $T_{0}$ and

$$
\begin{aligned}
\lim _{t \rightarrow T^{-}} & \left(\|u(t)\|^{2}+a \int_{0}^{t}\|u(s)\|^{2} d s+\gamma \int_{0}^{t}\left\|\left|\nabla_{g} u(s)\right|_{g}\right\|^{2} d s\right) \\
& =+\infty
\end{aligned}
$$

and then we have

$$
\lim _{t \rightarrow T^{-}}\left(\|u(t)\|^{2}+\left\|\left.\nabla_{g} u\right|_{g}\right\|^{2}\right)=+\infty .
$$

Case $2\left(E(0)=0\right.$ and $\left.\int_{\Omega} u_{0} u_{1} d x>0\right)$. By taking $r=0$ we have

$$
Q(t) \geq-p\left(E(0)+\frac{r}{2}\right)=0
$$

Furthermore, we have

$$
L(t) L^{\prime \prime}(t)-\frac{p+2}{4}\left(L^{\prime}(t)\right)^{2} \geq 0, \quad t \in\left[0, T_{0}\right] .
$$

Denoting $y(t)=L(t)^{-(p-2) / 4}$, we obtain that

$$
\begin{aligned}
y^{\prime \prime}(t) & =-\frac{p-2}{4} L(t)^{-(p+6) / 4}\left[L(t) L^{\prime \prime}(t)-\frac{p+2}{4}\left(L^{\prime}(t)\right)^{2}\right] \\
& \leq 0, \quad t \in\left[0, T_{0}\right] .
\end{aligned}
$$

Since $\int_{\Omega} u_{0} u_{1} d x>0$, then we have

$$
\left\|u_{0}\right\|^{2}>0, \quad L(0)>0 .
$$

Furthermore, we choose suitable positive constants $\tau$ and $T$ such that

$$
0<\frac{L(0)}{L^{\prime}(0)} \leq \frac{p-2}{4} T,
$$

and then from the fact that $L^{\prime}(0)>0$ we have

$$
L(t) \geq\left(\frac{4 L^{(p+2) / 4}(0)}{4 L(0)-(p-2) L^{\prime}(0) t}\right)^{4 /(p-2)},
$$

for some $t>0$. By the similar arguments as we did in the proof of Case 1, we can prove the desired limit.

Case $3\left(0<E(0)<E_{1}\right.$ and $\left.\left\|\mathscr{A} u_{0}\right\|>s_{1}\right)$. It follows from Lemma 3 that there exists $s_{2}>s_{1}$, such that

$$
\|\mathscr{A} u\| \geq s_{2}>s_{1} .
$$

Thus together with (110) and (124), it follows that

$$
\begin{aligned}
Q(t) & \geq-p E(0)+\frac{p-2}{2} s_{2}^{2}-\frac{p r}{2} \\
& =\left(-p E(0)+\frac{p-2}{2} s_{1}^{2}\right)+\left(\frac{p-2}{2}\left(s_{2}^{2}-s_{1}^{2}\right)-\frac{p r}{2}\right) .
\end{aligned}
$$

By taking $r=((p-2) / p)\left(s_{2}^{2}-s_{1}^{2}\right)>0$, we get

$$
Q(t) \geq 0, \quad L(0)>0 .
$$

Furthermore, we have

$$
L(t) L^{\prime \prime}(t)-\frac{p+2}{4}\left(L^{\prime}(t)\right)^{2} \geq 0, \quad t \in\left[0, T_{0}\right] .
$$


Denoting $y(t)=L(t)^{-(p-2) / 4}$, we obtain that

$$
\begin{aligned}
y^{\prime \prime}(t) & =-\frac{p-2}{4} L(t)^{-(p+6) / 4}\left[L(t) L^{\prime \prime}(t)-\frac{p+2}{4}\left(L^{\prime}(t)\right)^{2}\right] \\
& \leq 0, \quad t \in\left[0, T_{0}\right] .
\end{aligned}
$$

Furthermore, we choose suitable positive constants $\tau$ and $T$ such that

$$
0<\frac{L(0)}{L^{\prime}(0)} \leq \frac{p-2}{4} T
$$

and then we have

$$
L(t) \geq\left(\frac{4 L^{(p+2) / 4}(0)}{4 L(0)-(p-2) L^{\prime}(0) t}\right)^{4 /(p-2)},
$$

for some $t>0$. By the similar arguments as we did in the proof of Case 1, we can prove the desired limit. Theorem 6 is established.

\section{Global Existence Result}

In this section we give the result of the global existence of the solution to problem (1) which is similar to [26].

Theorem 7. Suppose $p, m$ satisfy (10)-(11) and $p \leq m$, the initial data $\left(u_{0}, u_{1}\right) \in W \times L^{2}(\Omega)$, and $u(x, t)$ is the solution of problem (1). Then the solution $u(x, t)$ is global.

Proof. Set

$$
P(t)=\frac{1}{2}\left\|u_{t}\right\|^{2}+\frac{1}{2}\|\mathscr{A} u\|^{2}+\frac{b}{p}\|u\|_{p}^{p} .
$$

After a simple computation, we get that

$$
P^{\prime}(t)=-a\left\|u_{t}\right\|_{m}^{m}-\gamma\left\|\left|\nabla_{g} u_{t}\right|_{g}\right\|^{2}+2 b \int_{\Omega}|u|^{p-2} u u_{t} d x .
$$

From Young's inequality, it follows that, for any $\delta>0$,

$$
P^{\prime}(t) \leq-a\left\|u_{t}\right\|_{m}^{m}-\gamma\left\|\left|\nabla_{g} u_{t}\right|_{g}\right\|^{2}+b \delta\left\|u_{t}\right\|_{p}^{p}+b C(\delta)\|u\|_{p}^{p} .
$$

Since $p \leq m$, then

$$
P^{\prime}(t) \leq-a\left\|u_{t}\right\|_{m}^{m}-\gamma\left\|\left|\nabla_{g} u_{t}\right|_{g}\right\|^{2}+b C \delta\left\|u_{t}\right\|_{m}^{p}+b C(\delta)\|u\|_{p}^{p},
$$

where $C>0$ is a constant depending on the domains $\Omega$ and $p$ and $C(\delta)>0$ is a constant depending on $\delta$. Then we get

$$
P^{\prime}(t) \leq c_{1}+c_{2} P(t) ;
$$

here and after in this section, $c_{i}(i=1,2,3)$ denote positive constants. Indeed, if $\left\|u_{t}\right\|_{m}>1$, then we can choose $\delta$ suitable small such that $-a\left\|u_{t}\right\|_{m}^{m}+b C \delta\left\|u_{t}\right\|_{m}^{p} \leq 0$, and hence

$$
P^{\prime}(t) \leq b C(\delta)\|u\|_{p}^{p} \leq p C(\delta) P(t)
$$

and otherwise, if $\left\|u_{t}\right\|_{m} \leq 1$, from (134), we obtain

$$
P^{\prime}(t) \leq b C \delta+b C(\delta)\|u\|_{p}^{p} \leq b C \delta+p C(\delta) P(t) .
$$

By integrating (135) we have, for $t \geq 0$,

$$
P(t) \leq\left(P(0)+c_{3}\right) e^{c_{2} t},
$$

where $c_{3}=c_{1} / c_{2}$. Theorem 7 is established.

\section{Global Asymptotic Stability Result}

In this section we establish the global asymptotic stability result to problem (1) with $b>0$ small enough and $p=2$ or $b=0$ (linear source term or no source term).

Theorem 8. Suppose $m$ satisfies (11), the initial data $\left(u_{0}, u_{1}\right) \in$ $W \times L^{2}(\Omega)$, and $u(x, t)$ be the solution of problem (1) with $0<$ $b<\lambda / B^{2}$ and $p=2$ or $b=0$ ( $B$ is the constant such that $\|u\| \leq$ $B\|\Delta u\|)$. Then the solution $u(x, t)$ is asymptotically stable, and the following decay estimate holds for $t>1$ sufficiently large:

$$
\begin{gathered}
\left\|u_{t}\right\|^{2}+\|\mathscr{A} u\|^{2}+\left\|\left.\nabla_{g} u\right|_{g}\right\|^{2} \leq C e^{-C t}, \quad m=2, \\
\left\|u_{t}\right\|^{2}+\|\mathscr{A} u\|^{2} \leq C t^{(1-m) / m}, \quad m \in(2,3), \\
\left\|u_{t}\right\|^{2}+\|\mathscr{A} u\|^{2} \leq C t^{-2 / m}, \quad m \geq 3,
\end{gathered}
$$

where the generic constant $C>0$ depends on $\Omega, \gamma, a, b, m$, and the initial data $\left(u_{0}, u_{1}\right)$.

Proof. For $0<b<\lambda / B^{2}$ and $p=2$ or $b=0$, using (48) with $p=2$ and (3), we have

$$
\begin{aligned}
E(0) & \geq E(t)=\frac{1}{2}\left\|u_{t}\right\|^{2}+\frac{1}{2}\|\mathscr{A} u\|^{2}-\frac{b}{2}\|u\|^{2} \\
& \geq \frac{1}{2}\left\|u_{t}\right\|^{2}+\left(\frac{1}{2}-\frac{b B^{2}}{2 \lambda}\right)\|\mathscr{A} u\|^{2} \\
& =: \frac{1}{2}\left\|u_{t}\right\|^{2}+\mu\|\mathscr{A} u\|^{2} \geq 0,
\end{aligned}
$$

where $b<\lambda / B^{2}$ and $\mu=1 / 2-b B^{2} / 2 \lambda$. Then thanks to (43), we have

$$
E(0)-E(t)=a \int_{0}^{t} \int_{\Omega}\left|u_{t}\right|^{m} d x d s+\gamma \int_{0}^{t} \int_{\Omega}\left|\nabla_{g} u_{t}\right|_{g}^{2} d x d s .
$$

Combining (140) and (141), we obtain

$$
E(0) \geq a \int_{0}^{t} \int_{\Omega}\left|u_{s}\right|^{m} d x d s+\gamma \int_{0}^{t} \int_{\Omega}\left|\nabla_{g} u_{s}\right|_{g}^{2} d x d s .
$$

Therefore, we get the following estimate:

$$
\begin{gathered}
\int_{0}^{t} \int_{\Omega}\left|u_{s}\right|^{m} d x d s \leq \frac{E(0)}{a}, \\
\int_{0}^{t} \int_{\Omega}\left|\nabla_{g} u_{s}\right|_{g}^{2} d x d s \leq \frac{E(0)}{\gamma} .
\end{gathered}
$$


Multiplying the equation of (1) by $u$ and integrating over $\Omega$, we obtain

$$
\begin{aligned}
& \frac{d}{d t} \int_{\Omega} u u_{t} d x-\left\|u_{t}\right\|^{2}+\|\mathscr{A} u\|^{2} \\
& \quad+\gamma \frac{d}{d t}\left\|\left|\nabla_{g} u\right|_{g}\right\|^{2}+a \int_{\Omega}\left|u_{t}\right|^{m-2} u_{t} u d x=b\|u\|_{2}^{2} .
\end{aligned}
$$

Multiplying (145) by a small enough positive constant $\delta$ to be determined and adding to the energy identity

$$
\begin{gathered}
\frac{d}{d t}\left[\frac{1}{2}\left\|u_{t}\right\|^{2}+\frac{1}{2}\|\mathscr{A} u\|^{2}-\frac{b}{p}\|u\|_{2}^{2}\right] \\
=-a\left\|u_{t}\right\|_{m}^{m}-\gamma\left\|\left|\nabla_{g} u_{t}\right|_{g}\right\|^{2},
\end{gathered}
$$

we get that

$$
\begin{aligned}
& \frac{d}{d t}\left[\frac{1}{2}\left\|u_{t}\right\|^{2}+\frac{1}{2}\|\mathscr{A} u\|^{2}-\frac{b}{2}\|u\|_{2}^{2}\right. \\
& \left.+\delta \int_{\Omega} u u_{t} d x+\delta \gamma\left\|\left|\nabla_{g} u\right|_{g}\right\|^{2}\right] \\
& =-a\left\|u_{t}\right\|_{m}^{m}-\gamma\left\|\left|\nabla_{g} u_{t}\right|_{g}\right\|^{2}+\delta\left\|u_{t}\right\|^{2}-\delta\|\mathscr{A} u\|^{2} \\
& \quad-a \delta \int_{\Omega}\left|u_{t}\right|^{m-2} u_{t} u d x+\delta b\|u\|_{2}^{2} .
\end{aligned}
$$

Set

$$
\begin{aligned}
J(t):= & \frac{1}{2}\left\|u_{t}\right\|^{2}+\frac{1}{2}\|\mathscr{A} u\|^{2}-\frac{b}{2}\|u\|_{2}^{2} \\
& +\delta \int_{\Omega} u u_{t} d x+\delta \gamma\left\|\left|\nabla_{g} u\right|_{g}\right\|^{2}
\end{aligned}
$$

Then the following inequality holds:

$$
\begin{aligned}
\frac{d}{d t} J(t)= & -a\left\|u_{t}\right\|_{m}^{m}-\gamma\left\|\left|\nabla_{g} u_{t}\right|_{g}\right\|^{2}+\delta\left\|u_{t}\right\|^{2} \\
& -\delta\|\mathscr{A} u\|^{2}-a \delta \int_{\Omega}\left|u_{t}\right|^{m-2} u_{t} u d x+\delta b\|u\|_{2}^{2} \\
\leq & -a\left\|u_{t}\right\|_{m}^{m}-\gamma\left\|\left|\nabla_{g} u_{t}\right|_{g}\right\|^{2}+\delta\left\|u_{t}\right\|^{2}-\delta\|\mathscr{A} u\|^{2} \\
& +a \delta \int_{\Omega}\left|u_{t}\right|^{m-1}|u| d x+\delta b\|u\|_{2}^{2} \\
= & 2 \delta\left\|u_{t}\right\|^{2}-a\left\|u_{t}\right\|_{m}^{m}-\gamma\left\|\left|\nabla_{g} u_{t}\right|_{g}\right\|^{2}-2 \delta E(t) \\
& +a \delta \int_{\Omega}\left|u_{t}\right|^{m-1}|u| d x .
\end{aligned}
$$

Integrating (149) with respect to $t$ over $(0, t)$, we obtain

$$
\begin{aligned}
2 \delta \int_{0}^{t} E(s) d s \leq & J(0)-J(t)+2 \delta \int_{0}^{t}\left\|u_{s}\right\|^{2} d s-a \int_{0}^{t}\left\|u_{s}\right\|_{m}^{m} d s \\
& -\gamma \int_{0}^{t}\left\|\left|\nabla_{g} u_{s}\right|_{g}\right\|^{2} d s \\
& +a \delta \int_{0}^{t} \int_{\Omega}\left|u_{s}\right|^{m-1}|u| d x d s .
\end{aligned}
$$

Combining the nondecreasing property of $E(t)$, we get the estimate

$$
\begin{aligned}
2 \delta t E(t) \leq & J(0)-J(t)+2 \delta \int_{0}^{t}\left\|u_{s}\right\|^{2} d s-a \int_{0}^{t}\left\|u_{s}\right\|_{m}^{m} d s \\
& -\gamma \int_{0}^{t}\left\|\left|\nabla_{g} u_{s}\right|_{g}\right\|^{2} d s+a \delta \int_{0}^{t} \int_{\Omega}\left|u_{s}\right|^{m-1}|u| d x d s .
\end{aligned}
$$

From Young's inequality and the definition of $J(t)$ and (140), we have

$$
\begin{aligned}
J(t) \geq & \frac{1}{2}\left\|u_{t}\right\|^{2}+\frac{1}{2}\|\mathscr{A} u\|^{2}-\frac{b}{2}\|u\|_{2}^{2}-\frac{\delta}{2}\|u\|^{2} \\
& -\frac{\delta}{2}\left\|u_{t}\right\|^{2}+\delta \gamma\left\|\left|\nabla_{g} u\right|_{g}\right\|^{2} \\
\geq & \left(\frac{1}{2}-\frac{\delta}{2}\right)\left\|u_{t}\right\|^{2}+\left(\mu-\frac{\delta B^{2}}{2 \lambda}\right)\|\mathscr{A} u\|^{2}+\delta \gamma\left\|\left|\nabla_{g} u\right|_{g}\right\|^{2} \\
\geq & \left(\frac{1}{2}-\frac{\delta}{2}\right)\left\|u_{t}\right\|^{2}+\left(\mu-\frac{\delta B^{2}}{2 \lambda}\right)\|\mathscr{A} u\|^{2}+\delta \gamma\left\|\left|\nabla_{g} u\right|_{g}\right\|^{2} \\
\geq & 0,
\end{aligned}
$$

for $\delta$ small enough. Therefore, the following estimate holds:

$$
\begin{aligned}
2 \delta t E(t) & \leq J(0)+2 \delta \int_{0}^{t}\left\|u_{s}\right\|^{2} d s+a \delta \int_{0}^{t} \int_{\Omega}\left|u_{s}\right|^{m-1}|u| d x d s \\
& =: J(0)+I_{1}+I_{2} .
\end{aligned}
$$

Using Hölder's inequality, (43), (140), and (143), we obtain

$$
\begin{aligned}
I_{1} & =2 \delta \int_{0}^{t} \int_{\Omega}\left|u_{s}\right|^{2} d x d s \\
& \leq 2 \delta\left(\int_{0}^{t} \int_{\Omega} 1 d x d s\right)^{(m-2) / m}\left(\int_{0}^{t} \int_{\Omega}\left|u_{s}\right|^{m} d x d s\right)^{2 / m} \\
& \leq 2 \delta\left(\frac{E(0)}{a}\right)^{2 / m}(C(\Omega))^{(m-2) / m} t^{(m-2) / m},
\end{aligned}
$$


where $C(\Omega)$ is a positive constant depending on $\Omega$, and

$$
\begin{aligned}
I_{2} & =a \delta \int_{0}^{t} \int_{\Omega}\left|u_{s}\right|^{m-1}|u| d x d s \\
& \leq a \delta\left(\int_{0}^{t} \int_{\Omega}\left|u_{s}\right|^{m} d x d s\right)^{(m-1) / m}\left(\int_{0}^{t} \int_{\Omega}|u|^{m} d x d s\right)^{1 / m} \\
& \leq a \delta C_{*}\left(\frac{E(0)}{a}\right)^{(m-1) / m}\left(\int_{0}^{t}\|\mathscr{A} u\|^{m} d s\right)^{1 / m} \\
& \leq a \delta C_{*}\left(\frac{E(0)}{a}\right)^{(m-1) / m}\left(\frac{E(0)}{\mu}\right)^{1 / 2} t^{1 / m}
\end{aligned}
$$

where $C_{*}$ is the constant such that $\|u\|_{m} \leq C_{*}\|\mathscr{A} u\|$. It follows from (153)-(155) that

$$
\begin{aligned}
2 \delta E(t) \leq & J(0) t^{-1}+2 \delta\left(\frac{E(0)}{a}\right)^{2 / m}(C(\Omega))^{(m-2) / m} t^{-2 / m} \\
& +a \delta C_{*}\left(\frac{E(0)}{a}\right)^{(m-1) / m}\left(\frac{E(0)}{\mu}\right)^{1 / 2} t^{(1-m) / m}
\end{aligned}
$$

Therefore, there are positive constants $K_{i}(i=1,2,3)$ such that

$$
E(t) \leq K_{1} t^{-1}+K_{2} t^{-2 / m}+K_{3} t^{(1-m) / m}
$$

Hence together with (140), we have, for $t>1$ sufficiently large, there exist positive constants $C>0$, such that

$$
\begin{aligned}
& \left\|u_{t}\right\|^{2}+\|\mathscr{A} u\|^{2} \leq C t^{(1-m) / m}, \quad m \in[2,3), \\
& \left\|u_{t}\right\|^{2}+\|\mathscr{A} u\|^{2} \leq C t^{-2 / m}, \quad m \geq 3 .
\end{aligned}
$$
$m=2$

In particular, in the case $m=2$, we obtain from (149) with

$$
\begin{aligned}
& \frac{d}{d t} J(t)+a\left\|u_{t}\right\|^{2}+\gamma\left\|\left|\nabla_{g} u_{t}\right|_{g}\right\|^{2}-\delta\left\|u_{t}\right\|^{2} \\
& +\delta\|\mathscr{A} u\|^{2}+a \delta \int_{\Omega} u_{t} u d x-\delta b\|u\|^{2}=0 .
\end{aligned}
$$

Multiplying $J(t)$ by a small enough positive constant $\delta^{*}$ to be determined and adding to (159), we obtain

$$
\begin{aligned}
0= & \frac{d}{d t} J(t)+a\left\|u_{t}\right\|^{2}+\gamma\left\|\left|\nabla_{g} u_{t}\right|_{g}\right\|^{2}-\delta\left\|u_{t}\right\|^{2} \\
& +\delta\|\mathscr{A} u\|^{2}+a \delta \int_{\Omega} u_{t} u d x-\delta b\|u\|^{2} \\
& +\delta^{*} J(t)-\frac{1}{2} \delta^{*}\left\|u_{t}\right\|^{2}-\frac{1}{2} \delta^{*}\|\mathscr{A} u\|^{2}+\frac{b}{2} \delta^{*}\|u\|^{2} \\
& -\delta \delta^{*} \int_{\Omega} u u_{t} d x-\delta \delta^{*} \gamma\left\|\left|\nabla_{g} u\right|_{g}\right\|^{2} \\
\geq & \frac{d}{d t} J(t)+\delta^{*} J(t)+\left(\frac{a}{2}-\delta-\delta^{*}\right)\left\|u_{t}\right\|^{2} \\
& +\gamma\left\|\left|\nabla_{g} u_{t}\right|_{g}\right\|^{2}+\left(\delta-\frac{\delta^{*}}{2}\right)\|\mathscr{A} u\|^{2}+\frac{\delta^{*} b}{2}\|u\|^{2} \\
& -\left(\delta b+\frac{\delta^{2} \delta^{*}}{2}+\frac{a \delta^{2}}{2}\right)\|u\|^{2}-\delta \delta^{*} \gamma\left\|\left|\nabla_{g} u\right|_{g}\right\|^{2} .
\end{aligned}
$$

Using (48) with $p=2$ and (3), we have

$$
\|u\|^{2} \leq \frac{B^{2}}{\lambda}\|\mathscr{A} u\|^{2} .
$$

It is not difficult to see that

$$
\left\|\left|\nabla_{g} u\right|_{g}\right\|^{2} \leq C\|\mathscr{A} u\|^{2}
$$

for some positive constant $C$. Together with (160)-(162), we can get the following estimate:

$$
\begin{aligned}
0 \geq & \frac{d}{d t} J(t)+\delta^{*} J(t)+\left(\frac{a}{2}-\delta-\delta^{*}\right)\left\|u_{t}\right\|^{2}+\gamma\left\|\left|\nabla_{g} u_{t}\right|_{g}\right\|^{2} \\
& +\left[\delta-\frac{\delta^{*}}{2}-\left(\delta b+\frac{\delta^{2} \delta^{*}}{2}+\frac{a \delta^{2}}{2}\right) \frac{B^{2}}{\lambda}-\delta \delta^{*} \gamma C\right]\|\mathscr{A} u\|^{2} \\
& +\delta b\|u\|^{2}
\end{aligned}
$$

We can choose $\delta$ and $\delta^{*}\left(\delta^{*}<\delta\right)$ sufficiently small with $0<$ $b<\lambda / B^{2}$ such that the following estimate holds:

$$
\frac{d}{d t} J(t)+\delta^{*} J(t) \leq 0
$$

Then we can get that

$$
J(t) \leq J(0) e^{-\delta^{*} t} .
$$

Thus due to (152), we have

$$
\left\|u_{t}\right\|^{2}+\|\mathscr{A} u\|^{2}+\left\|\left|\nabla_{g} u\right|_{g}\right\|^{2} \leq C e^{-C t},
$$

where the generic constants $C>0$ depend on $\Omega, \gamma, a, b$, and the initial data $\left(u_{0}, u_{1}\right)$. This completes the proof of Theorem 8. 


\section{Conflict of Interests}

The authors declare that there is no conflict of interests regarding the publication of this paper.

\section{Acknowledgments}

This work was partially supported by NNSF of China (61374089), NSF of Shanxi Province (2014011005-2), Shanxi Scholarship Council of China (2013-013), and Shanxi International Science and Technology Cooperation Projects (2014081026).

\section{References}

[1] H. A. Levine, "Instability and nonexistence of global solutions to nonlinear wave equations of the form $P u_{t t}=-A u+F(u)$," Transactions of the American Mathematical Society, vol. 192, pp. 1-21, 1974.

[2] H. A. Levine, "Some additional remarks on the nonexistence of global solutions to nonlinear wave equations," SIAM Journal on Mathematical Analysis, vol. 5, no. 1, pp. 138-146, 1974.

[3] V. K. Kalantarov and O. A. Ladyzhenskaya, "The occurrence of collapse for quasilinear equations of parabolic and hyperbolic types," Journal of Soviet Mathematics, vol. 10, no. 1, pp. 53-70, 1978.

[4] V. Georgiev and G. Todorova, "Existence of a solution of the wave equation with nonlinear damping and source terms," Journal of Differential Equations, vol. 109, no. 2, pp. 295-308, 1994.

[5] H. A. Levine and J. Serrin, "Global nonexistence theorems for quasilinear evolution equations with dissipation," Archive for Rational Mechanics and Analysis, vol. 137, no. 4, pp. 341-361, 1997.

[6] J. Hao, Y. Zhang, and S. Li, "Global existence and blow-up phenomena for a nonlinear wave equation," Nonlinear Analysis: Theory, Methods \& Applications, vol. 71, no. 10, pp. 4823-4832, 2009.

[7] S. A. Messaoudi, "Blow up in a nonlinearly damped wave equation," Mathematische Nachrichten, vol. 231, no. 1, pp. 1-7, 2001.

[8] S. A. Messaoudi and B. Said Houari, "Global non-existence of solutions of a class of wave equations with non-linear damping and source terms," Mathematical Methods in the Applied Sciences, vol. 27, no. 14, pp. 1687-1696, 2004.

[9] E. Vitillaro, "Global nonexistence theorems for a class of evolution equations with dissipation," Archive for Rational Mechanics and Analysis, vol. 149, no. 2, pp. 155-182, 1999.

[10] W. Chen and Y. Zhou, "Global nonexistence for a semilinear Petrovsky equation," Nonlinear Analysis: Theory, Methods \& Applications, vol. 70, no. 9, pp. 3203-3208, 2009.

[11] Y. Zhou, "Global nonexistence for a quasilinear evolution equation with critical lower energy," Archives of Inequalities and Applications, vol. 2, no. 1, pp. 41-47, 2004.

[12] P. Yao, "Observability inequalities for the Euler-Bernoulli plate with variable coefficients," in Differential Geometric Methods in the Control of Partial Differential Equations (Boulder, CO, 1999), vol. 268 of Contemporary Mathematics, pp. 383-406, American Mathematical Society, Providence, RI, USA, 2000.

[13] H. A. Levine, S. R. Park, and J. Serrin, "Global existence and global nonexistence of solutions of the Cauchy problem for a nonlinearly damped wave equation," Journal of Mathematical Analysis and Applications, vol. 228, no. 1, pp. 181-205, 1998.
[14] D. H. Sattinger, "On global solution of nonlinear hyperbolic equations," Archive for Rational Mechanics and Analysis, vol. 30, pp. 148-172, 1968.

[15] G. Todorova, "Cauchy problem for a nonlinear wave equation with nonlinear damping and source terms," Nonlinear Analysis: Theory, Methods \& Applications, vol. 41, no. 7-8, pp. 891-905, 2000.

[16] Y. Zhou, "Global existence and nonexistence for a nonlinear wave equation with damping and source terms," Mathematische Nachrichten, vol. 278, no. 11, pp. 1341-1358, 2005.

[17] Y. Zhou, "A blow-up result for a nonlinear wave equation with damping and vanishing initial energy in RN," Applied Mathematics Letters. An International Journal of Rapid Publication, vol. 18, no. 3, pp. 281-286, 2005.

[18] M. Köhl, "An extended Liapunov approach to the stability assessment of marine risers," Zeitschrift für Angewandte Mathematik und Mechanik, vol. 73, no. 2, pp. 85-92, 1993.

[19] V. K. Kalantarov and A. Kurt, "The long-time behavior of solutions of a nonlinear fourth order wave equation, describing the dynamics of marine risers," Zeitschrift für Angewandte Mathematik und Mechanik, vol. 77, no. 3, pp. 209-215, 1997.

[20] G. Avalos, I. Lasiecka, and R. Triggiani, "Uniform stability of nonlinear thermoelastic plates with free boundary conditions," in Optimal Control of Partial Differential Equations, K.-H. Hoffmann, G. Leugering, and F. Tröltzsch, Eds., Birkhäuser, Basel, Switzerland, 1999.

[21] A. Eden and A. J. Milani, "Exponential attractors for extensible beam equations," Nonlinearity, vol. 6, no. 3, pp. 457-479, 1993.

[22] A. Guesmia, "Existence globale et stabilisation interne non linéaire d'un système de Petrovsky," Bulletin of the Belgian Mathematical Society. Simon Stevin, vol. 5, no. 4, pp. 583-594, 1998.

[23] A. Guesmia, "Energy decay for a damped nonlinear coupled system," Journal of Mathematical Analysis and Applications, vol. 239, no. 1, pp. 38-48, 1999.

[24] K.-H. Hoffmann and P. Rybka, "Analyticity of the nonlinear term forces convergence of solutions to two equations of continuum mechanics," Nonlinear Analysis: Theory, Methods \& Applications, vol. 50, no. 3, pp. 409-424, 2002.

[25] J. Li and Y. Wu, "Exponential stability of the plate equations with potential of second order and indefinite damping," Journal of Mathematical Analysis and Applications, vol. 359, no. 1, pp. 6275, 2009.

[26] S. A. Messaoudi, "Global existence and nonexistence in a system of Petrovsky," Journal of Mathematical Analysis and Applications, vol. 265, no. 2, pp. 296-308, 2002.

[27] R. Xu and Y. Liu, "Global existence and nonexistence of solution for Cauchy problem of multidimensional double dispersion equations," Journal of Mathematical Analysis and Applications, vol. 359, no. 2, pp. 739-751, 2009.

[28] J. L. Lions, Quelques méthodes de résolution des problèmes aux limites non linéaires, Dunod Gautier-Villars, Paris, France, 1969. 


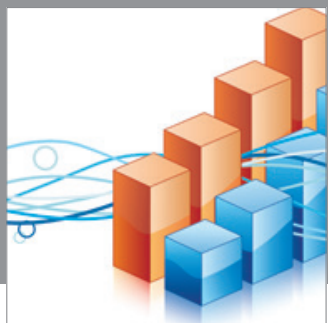

Advances in

Operations Research

mansans

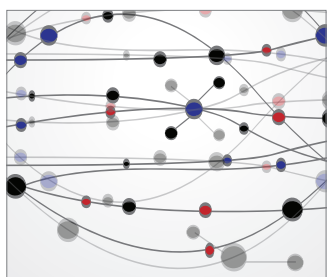

The Scientific World Journal
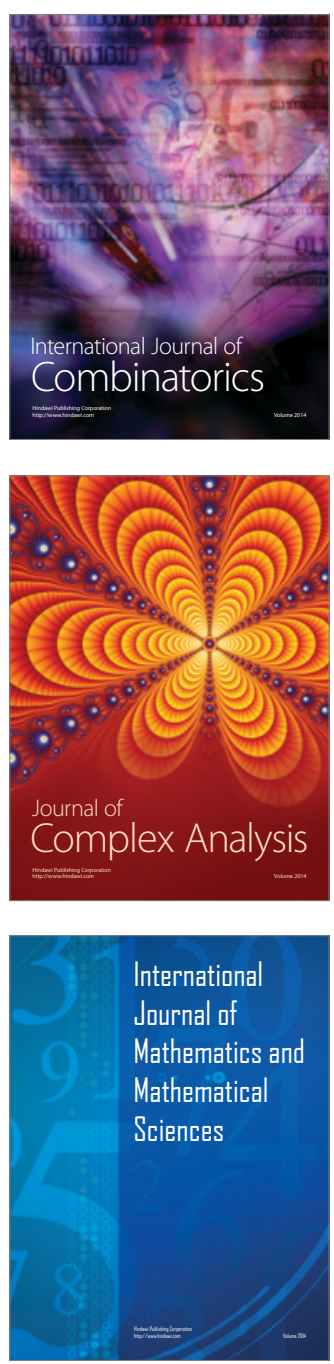
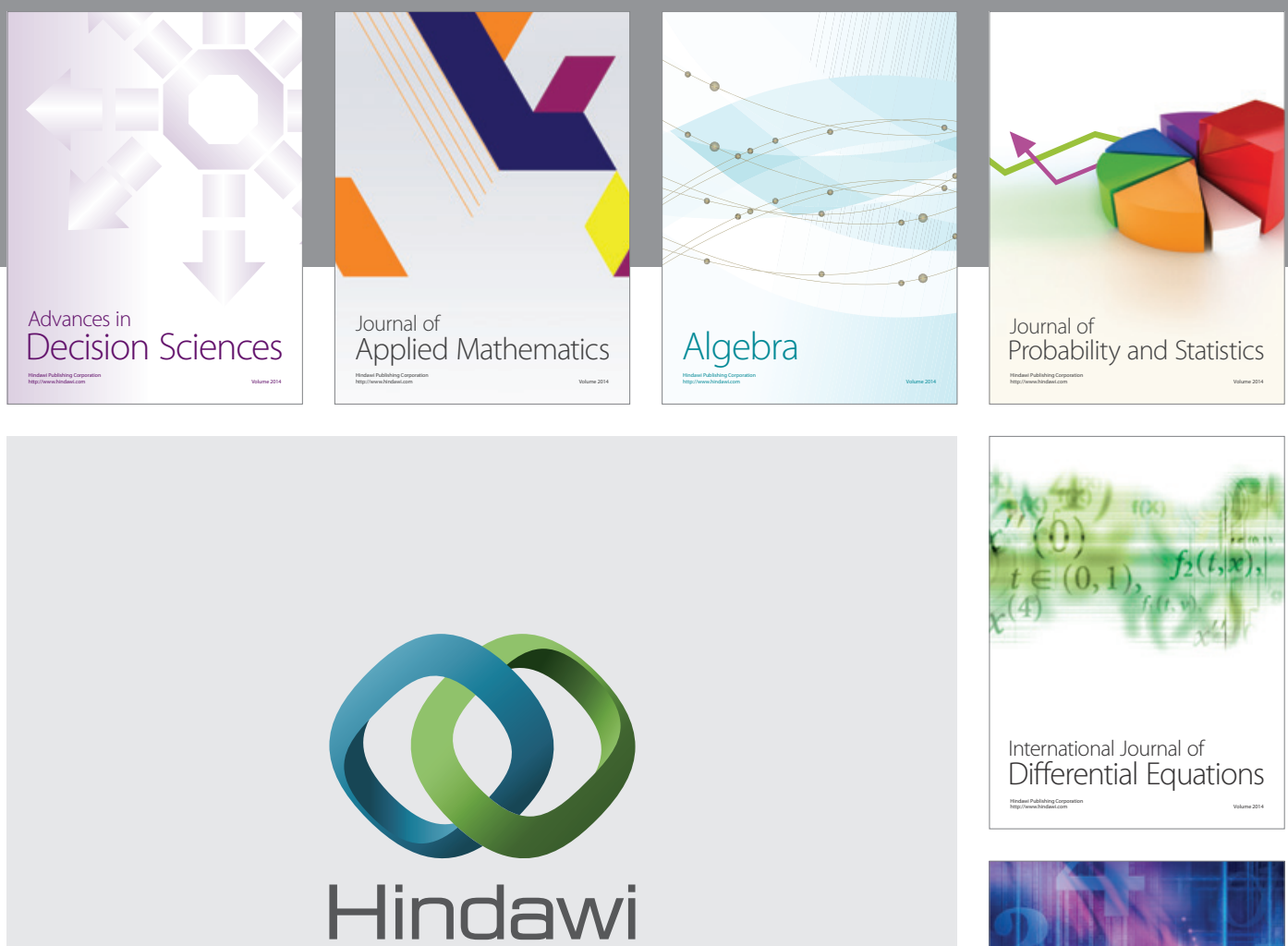

Submit your manuscripts at http://www.hindawi.com
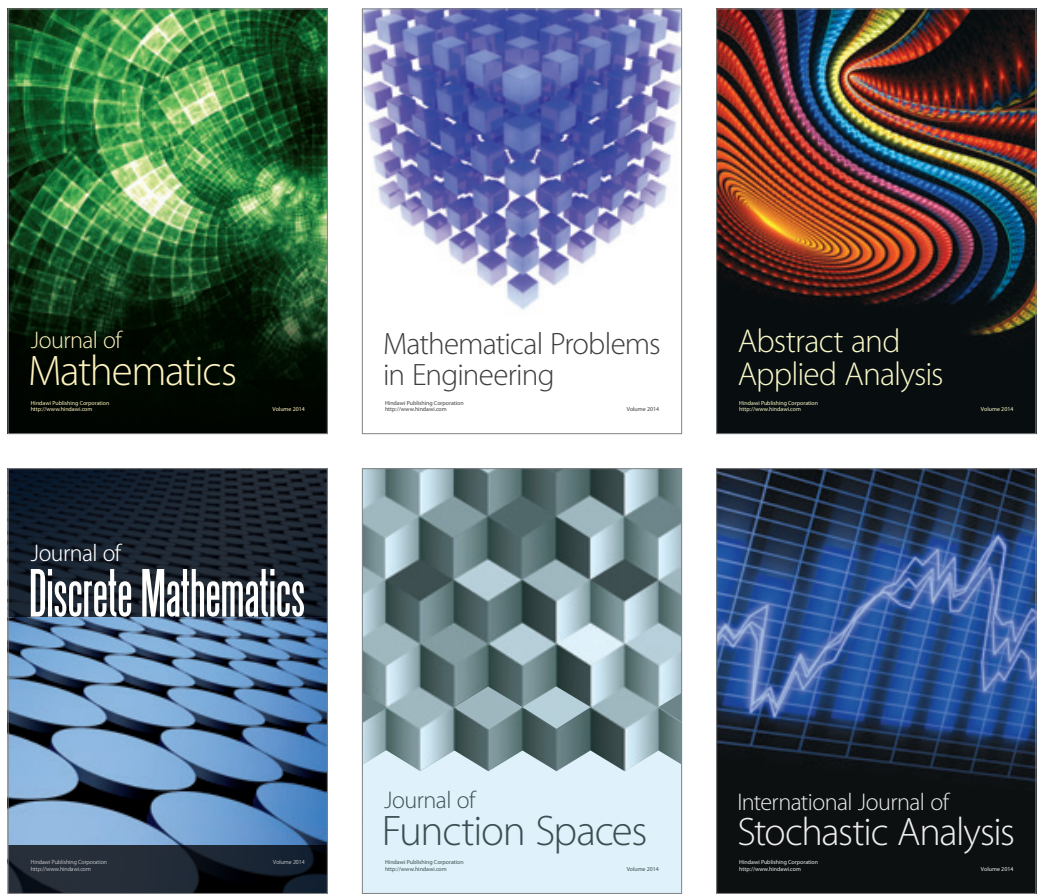

Journal of

Function Spaces

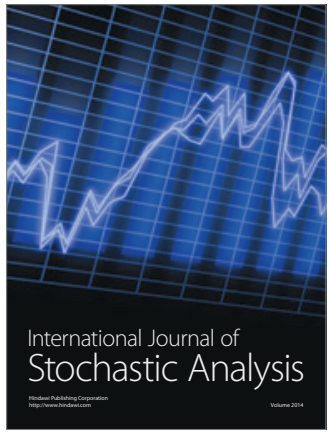

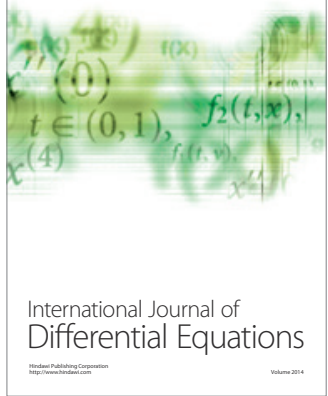
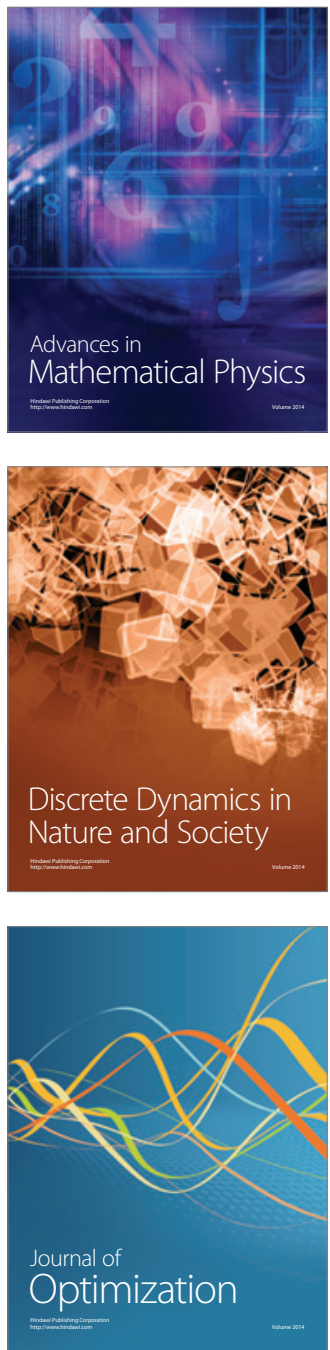